\title{
HARMONIC GAUSS SECTIONS, OBJECT INCLUSION MAPS AND YANG-MILLS CONNECTIONS
}

\author{
BY HIROKI MANABE
}

\section{Introduction}

Harmonic Gauss sections are critical points of the vertical energy through arbitrary vertical variations with respect to some fixed tangential splitting of a target Grassmann manifold bundle (definition in [11], [12]). On the other hand, Yang-Mills connections are critical points of the square norm of curvature form through arbitrary variations in the connection forms. There is a relationship between these variational solutions which is previously observed by C. M. Wood in [1],

Let $G$ be a Lie group which admits a bi-invariant Riemannian metric and $P$ be a right principal $G$-bundle over a Riemannian manifold $M$. The horizontally lifted metric on $P$ by a right connection form $\omega$ makes the fibration $\pi_{P}: P \rightarrow M$ into a Riemannian submersion with totally geodesic fibers [10]. The Yang-Mills equation for a single connection form $\omega$ is translated into the harmonic section equation for the Gauss section $\gamma_{P}=\left[P \ni u \mapsto \operatorname{Ker}\left(d \pi_{P}\right)_{u} \subset T_{u} P\right]$ on $P$ with the $\omega$-horizontally lifted metric [12]. In fact, C. M. Wood obtained:

THEOREM. Let $\omega$ be a right connection form on $P$. Then

(i) $\omega$ is flat (resp. Yang-Mills) if and only if

$\gamma_{P}$ is a horizontal (resp. harmonic) section with respect to ${ }^{P} \nabla$,

(ii) $\omega$ is parallel if $\gamma_{P}$ is a covariantly horizontal section with respect to ${ }^{P} \nabla$, where ${ }^{P} \nabla$ is the Riemannian connection of $P$.

In this paper we study several characterizations of Yang-Mills connections in terms of harmonic Gauss sections, each of which is a generalization of the theorem of C.M. Wood. Our results extend the lists of similarities between theories of harmonic maps and Yang-Mills connections by J. P. Bourguignon [1].

Let $Q$ be a left principal $G$-bundle over a Riemannian manifold $N$ and $\eta$ be a left connection form on $Q$, where the left $G$-action in the definition of left $\cdots$ is the reciprocal of the right $G$-action in that of right $\ldots$. The joint space $P \cdot Q$ (definition in $\S 1$ ) also has the horizontally lifted metric by the joint form $\omega \diamond \eta$ (definition in $\S 1$ ) which makes the fibration $\pi_{P \cdot Q}: P \cdot Q \rightarrow M \times N$ into

Received October 9, 1992 ; revised May 10, 1993. 
a Riemannian submersion with totally geodesic fibers.

A pair of the Yang-Mills equations for $\omega$ and $\eta$ is translated into the harmonic section equation for the Gauss section $\gamma_{P \cdot Q}=\left[P \cdot Q \ni u \cdot v \mapsto \operatorname{Ker}\left(d \pi_{P \cdot Q}\right)_{u \cdot v} \subset\right.$ $\left.T_{u \cdot v}(P \cdot Q)\right]$ on $P \cdot Q$ with the $\omega \diamond \eta$-horizontally lifted metric:

THEOREM A. Let $\omega, \eta$ be a right connection form on $P$ and a left connection form on $Q$, respectively. Then

(i) $\omega$ and $\eta$ are both flat (resp. Yang-Mills) if and only if

$\gamma_{P \cdot Q}$ is a horizontal (resp. harmonic) section with respect to ${ }^{P \cdot Q} \nabla$,

(ii) $\omega$ and $\eta$ are both parallel if

$\gamma_{P \cdot Q}$ is a covariantly horizontal section with respect to ${ }^{P \cdot Q} \nabla$, where ${ }^{P \cdot Q} \nabla$ is the Riemannian connection of $P \cdot Q$.

In the case of $N=M, Q=P^{-1}$ and $\eta=\omega^{-1}$, where $P^{-1}$ (resp. $\left.\omega^{-1}\right)$ is the inverse of $P$ (resp. $\omega$ ) [5], we have immediately the following:

COROLLARY B. Let $\omega$ be a right connection form on $P$. Then

(i) $\omega$ is flat (resp. Yang-Mills) if and only if

$\gamma_{P \cdot P-1}$ is a horizontal (resp. harmonic) section with respect to ${ }^{P \cdot P-1} \nabla$.

(ii) $\omega$ is parallel if

$\gamma_{P \cdot P-1}$ is a covariantly horizontal section with respect to ${ }^{P \cdot P-1} \nabla$.

By pulling-back $\gamma_{P \cdot P-1}$ along the object inclusion map $\varepsilon$ (definition in $\S 1$ ), the Gauss section ${ }^{\varepsilon} \gamma_{P \cdot P-1}=\left[M \ni x \mapsto \operatorname{Ker}\left(d \pi_{P \cdot P-1}\right)_{\varepsilon(x)} \subset T_{\varepsilon(x)}\left(P \cdot P^{-1}\right)\right]$ is induced and the above relation (ii) is improved (Theorems $4.7,4.14,4.16)$ :

THEOREM C. Let $\omega$ be a right connection form on $P$. Then $\omega$ is flat, parallel or Yang-Mills if and only if

${ }^{8} \gamma_{P \cdot P-1}$ is a horizontal, covariantly horizontal or harmonic section with respect to ${ }^{\varepsilon}\left(P^{P \cdot P-1} \nabla\right)$, respectively,

where $\left.{ }^{\varepsilon\left(P \cdot P^{-1}\right.} \nabla\right)$ is the induced connection from ${ }^{P \cdot P^{-1}} \nabla$ via $\varepsilon$.

$\varepsilon(M)$ is a totally geodesic submanifold of $P \cdot P^{-1}$ (Proposition 2.16) so that ${ }^{\varepsilon}\left(P^{P \cdot P^{-1}} \nabla\right)$ splits into the Riemannian connection ${ }^{M} \nabla$ of $M$ and the normal connection $\nabla^{(-)}$with respect to $\varepsilon$. On the other hand, ${ }^{\varepsilon} \gamma_{P \cdot P-1}$ can be reduced to ${ }^{\varepsilon} \gamma_{P, P-1}^{(-)}=\left[M \ni x \mapsto \operatorname{Ker}\left(d \pi_{P, P-1}\right)_{\varepsilon(x)} \subset \boldsymbol{E}_{\varepsilon(x)}^{(-)}\right]$, where $\boldsymbol{E}_{\varepsilon(x)}^{(-)}$is the -1-eigenspace of the differential of the inversion of $P \cdot P^{-1}$, which is also the orthogonal complement of $(d \varepsilon)_{x} T_{x} M$ in $T_{\varepsilon(x)}\left(P \cdot P^{-1}\right)$.

THEOREM D (Theorem 5.12). Let $\omega$ be a right connection form on $P$. Then $\omega$ is flat, parallel or Yang-Mills if and only if

${ }^{8} \gamma_{P \cdot P-1}^{(-)}$is a horizontal, covariantly horizontal or harmonic section with respect to $\nabla^{(-)}$, respectively. 
Acknowledgement. The author wishes to express his sincere thanks to professor Akira Asada for leading our attentions to Lie groupoids, to professor Hisao Nakagawa and professor Tsunero Takahashi for their hearty guidance and many valuable suggestions and to the referee for his helpful comments.

\section{§1. Joints of Principal Bundles and Connection Forms}

Let $G$ be a Lie group and $P \stackrel{\pi_{P} P}{\rightarrow} M$ (resp. $Q \stackrel{\pi_{Q}}{\rightarrow} N$ ) be a right (resp. left) principal $G$-bundle over a $C^{\infty}$-manifold $M$ (resp. $\left.N\right) . G$ acts on $P \times Q$ as follows ; ${ }^{P \times Q} R: P \times Q \times G \rightarrow P \times Q ;((u, v), g) \mapsto\left(u g, g^{-1} v\right)$ (resp. ${ }^{P \times Q} L: G \times P \times Q \rightarrow$ $P \times Q ;(g,(u, v)) \mapsto\left(u g^{-1}, g v\right)$.) We denote the quotient topological space of the right (resp. left) $G$-space $\left(P \times Q,{ }^{P \times Q} R\right)$ (resp. $\left.\left(P \times Q,{ }^{P \times Q} L\right)\right)$ by $P \cdot Q$ and the quotient map by $j_{P \cdot Q}: P \times Q \rightarrow P \cdot Q ;(u, v) \mapsto u \cdot v$. The map $\pi_{P \cdot Q}: P \cdot Q \rightarrow M \times N$; $u \cdot v \mapsto\left(\pi_{P}(u), \pi_{Q}(v)\right)$ is well-defined. By [3, Chapter 5, Section 2, Proposition 1], $P \cdot Q$ is given a $C^{\infty}$-manifold structure (which is called the joint space of $P$ and $Q$ ).

$\left(P \times Q,{ }^{P \times Q} R\right) \stackrel{J_{P} \cdot Q}{\longrightarrow} P \cdot Q$ (resp. $\left.\left(P \times Q,{ }^{P \times Q} L\right) \stackrel{P^{P} \cdot Q}{\longrightarrow} P \cdot Q\right)$ is a right (resp. left) principal $G$-bundle. $P \cdot Q \stackrel{\pi_{P} \cdot Q}{\longrightarrow} M \times N$ is a $C^{\infty}$-fiber bundle. The canonical projection $p_{P}:\left(P \times Q,{ }^{P \times Q} R\right) \rightarrow P ;(u, v) \mapsto u \quad\left(\right.$ resp. $\left.p_{Q}:\left(P \times Q,{ }^{P \times Q} L\right) \rightarrow Q ;(u, v) \mapsto v\right)$ is a right (resp. left) principal $G$-bundle homomorphism. Throughout the following sections, $\left(P \times Q,{ }^{P \times Q} R\right)$ will be simply abbreviated to $P \times Q$. Let $\left(G, \operatorname{Ad}_{G}, \mathfrak{g}\right)$ be the adjoint representation of $G$. $\operatorname{Ker} d \pi_{P}$ (resp. $\operatorname{Ker} d \pi_{Q}$ ) is isomorphic to the trivial vector bundle $P \times g$ (resp. $g \times Q$ ). Similarly we observe the following:

\section{PROPOSITION 1.1.}

$$
\begin{aligned}
& \operatorname{Ker} d j_{P \cdot Q} \longrightarrow P \times Q \times \mathfrak{g} ; A_{(u, v)}^{\#}=\left(A_{u}^{\#},-A_{v}^{b}\right) \mapsto((u, v), A) \text { and } \\
& \operatorname{Ker} d \pi_{P \cdot Q} \longrightarrow(P \times Q)_{\operatorname{Ad}_{G}} g ;\left(d j_{P \cdot Q}\right)_{(u, v)}\left(A_{u}^{\#}, A_{v}^{b}\right) \mapsto[(u, v), A]
\end{aligned}
$$

are both vector bundle isomorphisms,

where $A_{u}^{\#}=d /\left.d t\right|_{t=0} u(\exp t A), A_{v}^{b}=d /\left.d t\right|_{t=0}(\exp t A) v(A \in \mathfrak{g})$ e.t.c..

Let $\omega$ (resp. $\eta$ ) be a right (resp. left) connection from on $P$ (resp. $Q$ ). Then from Proposition 1.1, $p_{P}^{*} \omega$ and $-p_{Q}^{*} \eta$ are both right connection forms on $P \times Q$ and therefore a tensorial form of type $\left(G, \operatorname{Ad}_{G}, g\right)$ (c. f. [6]).

Proposition 1.2. Let $\theta \in \boldsymbol{R}$.

(i) $(1-\theta) p_{P}^{*} \omega-\theta p_{Q}^{*} \eta$ is a right connection form on $P \times Q$.

(ii) $(1-\theta) p_{P}^{*} \omega+\theta p_{Q}^{*} \eta$ is a tensorial 1-form on $P \times Q$ of type $\left(G, \operatorname{Ad}_{G}, g\right)$ if and only if $\theta=1 / 2$.

We write shortly $\omega \star \eta$ instead of $1 / 2\left(p_{P}^{*} \omega-p_{Q}^{*} \eta\right)$. On the other hand, from 
the above proposition, $1 / 2\left(p_{P}^{*} \omega+p_{Q}^{*} \eta\right)$ can be reduced to a 1 -form on $P \cdot Q$ taking values in $(P \times Q) \times \operatorname{Ad}_{G} \mathfrak{g}$, which is denoted by $\omega \diamond \eta$ (which is called the joint form of $\omega$ and $\eta)$.

For any $u \in P, \quad v \in Q, \operatorname{Ker}(\omega \star \eta)_{(u, v)}=\left(\operatorname{Ker} \omega_{n} \oplus 0_{v}\right) \oplus\left\{\left(A_{u}^{\#}, A_{v}^{b}\right) \mid A \in \mathfrak{g}\right\} \oplus\left(0_{u} \oplus\right.$ $\left.\operatorname{Ker} \eta_{v}\right)$, where $\left(\operatorname{Ker} \omega_{u} \oplus 0_{v}\right) \oplus\left(0_{u} \oplus \operatorname{Ker} \eta_{v}\right)$ is the $\omega \star \eta$-horizontal lift of $\operatorname{Ker}(\omega \diamond \eta)_{u \cdot v}$ and $\left\{\left(A_{u}^{\#}, A_{v}^{b}\right) \mid A \in g\right\}$ is the $\omega \star \eta$-horizontal lift of $\operatorname{Ker}\left(d \pi_{P \cdot Q}\right)_{u \cdot v \cdot}$. On the other hand, Ker $\omega \diamond \eta$ is a horizontal distribution relative to $\pi_{P \cdot Q}$ :

Proposition 1.3. $T(P \cdot Q)=\operatorname{Ker} d \pi_{P \cdot Q} \oplus \operatorname{Ker} \omega \diamond \eta$.

Proof. For each $u \in P, v \in Q$, the restriction of surjective linear map $(\omega \diamond \eta)_{u \cdot v}: T_{u \cdot v}(P \cdot Q) \rightarrow\left((P \times Q) \times_{\operatorname{Ad}_{G}} g\right)_{u \cdot v}$ to $\operatorname{Ker}\left(d \pi_{P \cdot Q}\right)_{u \cdot v}$ coincides with the linear isomorphism in Proposition 1.1 .

The above proposition implies that $\omega \diamond \eta$ plays the same role of a connection form. Let ${ }^{\omega \star \eta} d$ (resp. ${ }^{\omega \star \eta} D$ ) be the exterior covariant differentiation on $P \cdot Q$ (resp. $P \times Q$ ) with respect to $\omega \star \eta$.

Proposition 1.4. For any $u \in P, v \in Q, X, Y \in \operatorname{Ker}(\omega \diamond \eta)_{u \cdot v}$,

$$
\left({ }^{\omega \star \eta} d(\omega \diamond \eta)\right)_{u \cdot v}(X, Y)=\left[(u, v), 1 / 2\left(\left(p_{P}^{*}\right)^{\omega} \Omega+\left(p_{Q}^{*}\right)^{\eta} \Omega\right)\left({ }^{\omega \star \eta} H_{u, v}^{(u, v)} X,{ }^{\omega} \star \eta H_{u, v}^{(u, v)} Y\right)\right],
$$

where ${ }^{\omega} \Omega\left(\right.$ resp. $\left.{ }^{\eta} \Omega\right)$ is the curvature form of $\omega$ (resp. $\left.\eta\right)$ and ${ }^{\omega \star \eta} H_{u, v}^{(u, v)}: T_{u \cdot v}(P \cdot Q)$ $\rightarrow \operatorname{Ker}(\omega \star \eta)_{(u, v)}$ is the $\omega \star \eta$-horizontal lifting.

Proof. By the structure equations for $\omega$ and $\eta$ (c. f. [6]),

$$
\begin{aligned}
& { }^{P \times Q} d\left(\frac{1}{2}\left(p_{P}^{*} \omega+p_{Q}^{*} \eta\right)\right)=\frac{1}{2}\left(\left(p_{P}^{*}\right)^{P} d \omega+\left(p_{Q}^{*}\right)^{Q} d \eta\right) \\
& =\frac{1}{2}\left\{p_{P}^{*}\left(\omega \Omega+\frac{1}{2}[\omega \wedge \omega]\right)+p_{Q}^{*}\left(\eta \Omega-\frac{1}{2}[\eta \wedge \eta]\right)\right\} \\
& =\frac{1}{2}\left(\left(p_{P}^{*}\right)^{\omega} \Omega+\left(p_{Q}^{*}\right)^{\eta} \Omega\right)-\frac{1}{4}\left(\left[p_{P}^{*} \omega \wedge p_{P}^{*} \omega\right]-\left[p_{Q}^{*} \eta \wedge p_{Q}^{*} \eta\right]\right) \\
& =\frac{1}{2}\left(\left(p_{P}^{*}\right)^{\omega} \Omega+\left(p_{Q}^{*}\right)^{\eta} \Omega\right)-\left[\frac{1}{2}\left(p_{P}^{*} \omega-p_{Q}^{*} \eta\right) \wedge \frac{1}{2}\left(p_{P}^{*} \omega+p_{Q}^{*} \eta\right)\right] .
\end{aligned}
$$

$1 / 2\left(\left(p_{P}^{*}\right)^{\omega} \Omega+\left(p_{Q}^{*}\right)^{\eta} \Omega\right)$ is a tensorial 2 -form on $P \times Q$ so that ${ }^{\omega \star \eta} D\left(1 / 2\left(p_{P}^{*} \omega+p_{Q}^{*} \eta\right)\right)$ $=1 / 2\left(\left(p_{P}^{*}\right)^{\omega} \Omega+\left(p_{Q}^{*}\right)^{\eta} \Omega\right)$.

COROLLARY 1.5. $\omega$ and $\eta$ are both flat if and only if $\operatorname{Ker} \omega \diamond \eta$ is an involutive distribution on $P \cdot Q$.

In the case of $N=M, Q=P^{-1}=P \times{ }_{\lambda} G$ which is called the inverse of $P$ in [5] defined by the left $G$-action $\lambda: G \times G \rightarrow G ;\left(g_{1}, g_{2}\right) \mapsto g_{2} g_{1}^{-1}$, for the diffeomorphism $i: P \rightarrow P^{-1} ; u \mapsto u^{-1}=[u, e]$, we denote $-\left(i^{-1}\right)^{*} \omega$ by $\omega^{-1}$ which is a left connection form on $P^{-1}$, where $e$ is the identity element of $G$. Note that $\omega^{-1} \Omega=-\left(i^{-1}\right)^{* \omega} \Omega$. 
COROLLARY 1.6. $\omega$ is flat if and only if $\operatorname{Ker} \omega \diamond \omega^{-1}$ is an involutrve distribution on $P \cdot P^{-1}$.

$P \cdot P^{-1}$ is the Lie groupoid associated to $P \stackrel{\pi_{P}}{\rightarrow} M$ and $\pi_{P \cdot P-1}: P \cdot P^{-1} \rightarrow M \times M$ is the anchor of $P \cdot P^{-1}\left[8\right.$, Chapter 1, Example 1.10]. Let $\Delta_{M}: M \rightarrow M \times M$ be the diagonal map of $M$ and $\Delta_{M}^{-1}\left(P \cdot P^{-1}\right)$ be the pull-back fiber bundle over $M$ via $\Delta_{M}$ which is the inner subgroupoid of $P \cdot P^{-1}$.

$\Delta_{M}^{-1}\left(P \cdot P^{-1}\right)$ is isomorphic to the automorphism bundle $P \times_{\mathrm{Ad}} G$. In fact, because $\Delta_{M}^{-1}\left(P \cdot P^{-1}\right)$ can be identified with $\left\{\left(x, u \cdot v^{-1}\right) \in M \times P \cdot P^{-1} \mid \pi_{P}(u)=x=\right.$ $\left.\pi_{P}(v)\right\}$, therefore, the following map is well-defined and gives a fiber bundle isomorphism ; $\Delta_{M}^{-1}\left(P \cdot P^{-1}\right) \rightarrow P \times_{\mathrm{Ad}} G ;\left(x, u \cdot v^{-1}\right) \mapsto[u, g](v=u g, g \in G)$.

Let $\tilde{\Delta}_{M}: P \times_{\mathrm{Ad}} G \rightarrow P \cdot P^{-1}$ be the induced fiber bundle homomorphism from $\Delta_{M}$ and $e: M \rightarrow P \times_{\text {Ad }} G$ be the canonical section which assigns $x$ to the identity element $e_{x}$ of the fiber over $x$, then their composite $\varepsilon=\tilde{\Delta}_{M^{\circ}} e: M \rightarrow P \cdot P^{-1}$ is a $C^{\infty}$-map, which is called the object inclusion map of $P \cdot P^{-1}$ and $\pi_{P \cdot P-1} \circ \varepsilon=\Delta_{M}$.

Let $\varepsilon^{-1}\left(P \times P^{-1}\right)$ be the pull-back principal $G$-bundle over $M$ via $\varepsilon \cdot \varepsilon^{-1}\left(P \times P^{-1}\right)$ is isomorphic to $P$. Actually we have a principal bundle homomorphism $\tilde{\varepsilon}: P \rightarrow P \times P^{-1} ; u \mapsto\left(u, u^{-1}\right)$ such that $j_{P \cdot P-1} \circ \tilde{\varepsilon}=\varepsilon \circ \pi_{P}$ and $\tilde{\varepsilon}^{*}\left(\omega \star \omega^{-1}\right)=\omega$. Because for $x \in M, u, v \in P, \varepsilon(x)=u \cdot v^{-1}$ if and only if $u=v \in \pi_{P}^{-1}(x)$, then $\varepsilon^{-1}\left(P \times P^{-1}\right)$ can be identified with $\left\{\left(x,\left(u, u^{-1}\right)\right) \in M \times P \times P^{-1} \mid u \in \pi_{P}^{-1}(x)\right\}$ so that the map $\varepsilon^{-1}\left(P \times P^{-1}\right) \rightarrow P ;\left(x,\left(u, u^{-1}\right)\right) \mapsto u$ is well-defined and gives a right principal $G$ bundle isomorphism.

PROPOSITION 1.7. $\varepsilon^{-1}\left(P \times P^{-1}\right) \times{ }_{\mathrm{Ad}_{G}} \mathrm{~g}$ is isomorphic to the adjoint bundle $P \times{ }_{\mathrm{Ad}_{G}} \mathrm{~g}$.

The notion of general groupoid includes its inversion. In the case of $P \cdot P^{-1}$, its inversion is $\iota: P \cdot P^{-1} \rightarrow P \cdot P^{-1} ; u \cdot v^{-1} \mapsto v \cdot u^{-1}$, which is obviously welldefined and $\iota^{\circ} \iota=$ id. $P \cdot P-1, \varepsilon$ is also given by $\varepsilon(x)=u \cdot u^{-1}\left(u \in \pi_{P}^{-1}(x)\right)$ so that $\iota \circ \varepsilon=\varepsilon$, therefore, the differential $d \iota: T\left(P \cdot P^{-1}\right) \rightarrow T\left(P \cdot P^{-1}\right)$ induces a vector bundle involutive automorphism of $\varepsilon^{-1} T\left(P \cdot P^{-1}\right)$ and a splitting $\left(\varepsilon^{-1} T\left(P \cdot P^{-1}\right)\right)_{x}$ $=T_{\varepsilon(x)}\left(P \cdot P^{-1}\right)=\boldsymbol{E}_{\varepsilon(x)}^{(+)} \oplus \boldsymbol{E}_{\varepsilon(x)}^{(-)}$, for each $x \in M$, where $\boldsymbol{E}_{\varepsilon(x)}^{(+)}$is the \pm 1 -eigenspace of $(d \iota)_{\varepsilon(x)} \cdot \varepsilon^{-1} \boldsymbol{E}^{( \pm)}=\prod_{x \in M} \boldsymbol{E}_{\varepsilon(x)}^{( \pm)}$is a vector subbundle of $\varepsilon^{-1} T\left(P \cdot P^{-1}\right)$ and $\varepsilon^{-1} T\left(P \cdot P^{-1}\right)$ $=\varepsilon^{-1} \boldsymbol{E}^{(+)} \oplus \boldsymbol{\varepsilon}^{-1} \boldsymbol{E}^{(-)}$.

Let ${ }^{\omega} H_{x}^{u}$ (resp. $\left.{ }^{\omega^{-1}} H_{x}^{u-1}\right): T_{x} M \rightarrow \operatorname{Ker} \omega_{u}\left(\operatorname{resp} . \operatorname{Ker} \omega_{u}^{-1}\right)$ be the $\omega$-(resp. $\omega^{-1}-$ ) horizontal lifting. Note that for $u \in \pi_{P}^{-1}(x), g \in G, X \in T_{x} M$,

$$
\begin{gathered}
\left(d j_{P \cdot P-1}\right)_{\left(u g, g^{-1} u-1\right)}\left({ }^{\omega} H_{x}^{u g} X, \pm{ }^{\omega^{-1}} H_{x}^{g^{-1} u-1} X\right) \\
=\left(d j_{P \cdot P-1}\right)_{(u, u-1)}\left({ }^{\omega} H_{x}^{u} X, \pm{ }^{\omega^{-1}} H_{x}^{u} X\right) .
\end{gathered}
$$

We write shortly ${ }^{\omega \diamond \omega^{-1}} X^{( \pm)}$instead of $\left(d_{P \cdot P-1}\right)_{(u, u-1)}\left({ }^{\omega} H_{x}^{u} X, \pm{ }^{\omega^{-1}} H_{x}^{u-1} X\right)$. 
Proposition 1.8. For any $x \in M$,

(i ) $(d \varepsilon)_{x}(X)={ }^{\omega \diamond \omega^{-1}} X^{(+)}$for all $X \in T_{x} M$, therefore,

$$
(d \varepsilon)_{x}\left(T_{x} M\right)=\boldsymbol{E}_{\varepsilon(x)}^{(+)}=\left\{\omega \diamond \omega^{-1} X^{(+)} \mid X \in T_{x} M\right\},
$$

(ii) $\boldsymbol{E}_{\varepsilon(x)}^{(-)}=\operatorname{Ker}\left(d \pi_{P \cdot P-1}\right)_{\varepsilon(x)} \oplus\left\{{ }^{\omega \diamond \omega^{-1}} X^{(-)} \mid X \in T_{x} M\right\}$,

(iii) $\operatorname{Ker}\left(\omega \diamond \omega^{-1}\right)_{\varepsilon(x)}=(d \varepsilon)_{x}\left(T_{x} M\right) \oplus\left\{\omega \diamond \omega^{-1} X^{(-)} \mid X \in T_{x} M\right\}$.

COROLLARY 1.9. For any $\omega, \varepsilon^{*}\left(\omega \diamond \omega^{-1}\right)=0$, therefore, $\varepsilon$ is an $\omega \diamond \omega^{-1}$-horizontal lift of $\Delta_{M}$.

\section{$\S 2$. Horizontally Lifted Metrics by the Joint Forms}

In this section, we assume that a Lie group $G$ admits a bi-invariant Riemannian metric $\langle$, $\rangle$. Let $M$ (resp. $N$ ) be a Riemannian manifold with a Riemannian metric ${ }^{M} g$ (resp. ${ }^{N} g$ ). For a right (resp. left) principal $G$-bundle $P \stackrel{\pi}{\rightarrow} M\left(\right.$ resp. $Q \stackrel{\pi_{Q}}{\rightarrow} N$ ) with a right (resp. left) connection form $\omega$ (resp. $\eta$ ), the $\omega$-(resp. $\eta$-)horizontally lifted metric ${ }_{\omega}^{P} g$ (resp. ${ }_{\eta}^{Q} g$ ) is defined as follows:

$$
{ }_{\omega}^{P} g=\left(\pi_{P}^{*}\right)^{M} g+\langle\boldsymbol{\omega}, \boldsymbol{\omega}\rangle \quad\left(\text { resp. }{ }_{\eta}^{Q} g=\left(\pi_{Q}^{*}\right)^{N} g+\langle\eta, \eta\rangle\right) .
$$

$\pi_{P}:(P, \stackrel{P}{\omega} g) \rightarrow\left(M,{ }^{M} g\right)$ (resp. $\left.\pi_{Q}:\left(Q,{ }_{\eta}^{Q} g\right) \rightarrow\left(N,{ }^{N} g\right)\right)$ is a Riemannian submersion with totally geodesic fibers since the $\omega$-(resp. $\eta$-) parallel translations between fibers are isometries in the above metric [10, Theorem 3.5]. We denote the canonical projection by $p_{M}\left(\right.$ resp. $\left.p_{N}\right): M \times N \rightarrow M($ resp. $N) ;(x, y) \mapsto \omega$ (resp. $\left.y\right)$. The joint form $\omega \diamond \eta$ lifts the Riemannian product metric ${ }^{M \times N} g=\left(p_{M}^{*}\right)^{M} g+\left(p_{N}^{*}\right)^{N} g$ to the metric $\underset{\omega \diamond \diamond \cdot Q}{P \cdot Q}$ on $P \cdot Q$ defined by ${ }_{\omega \diamond \eta}^{P \cdot Q} g=\left(\pi_{P \cdot Q}^{*}\right)^{M \times N} g+2\langle\omega \diamond \eta, \omega \diamond \eta\rangle$. From $\left[10, \quad\right.$ Theorem 3.5], we have $\pi_{P}^{Q}:\left(P \cdot Q, \stackrel{P}{\omega_{\omega \diamond} \cdot Q} g\right) \rightarrow\left(M,{ }^{M} g\right) ; u \cdot v \mapsto \pi_{P}(u), \pi_{Q}^{P}$ : $(P \cdot Q, \stackrel{P \cdot Q}{\omega \diamond \eta} g) \rightarrow\left(N,{ }^{N} g\right) ; u \cdot v \mapsto \pi_{Q}(v)$ and $\pi_{P \cdot Q}:(P \cdot Q, \underset{P \cdot Q}{P \diamond \eta} g) \rightarrow\left(M \times N,{ }^{M \times N} g\right)$ are Riemannian submersions with totally geodesic fibers.

Let ${ }^{P \times Q} g=\left(p_{P}^{*}\right)_{\omega}^{P} g+\left(p_{Q}^{*}\right)_{\eta}^{Q} g$ be the Riemannian product metric on $P \times Q$. Then ${ }^{P \times Q} g$ coincides with the $\omega \star \eta$-horizontally lifted metric from $\underset{\omega \diamond \eta}{P \cdot Q} g$ :

PROPOSITION 2.1. ${ }^{P \times Q} g=\left(j_{P}^{*} \cdot Q\right)_{\omega \diamond \eta}^{P \cdot Q} g+2\langle\omega \star \eta, \omega \star \eta\rangle$.

Throughout the following sections, the $\omega \star \eta$-horizontally lifted vector ${ }^{\omega \diamond \eta} H_{u \cdot v}^{(u, v)} U$ will be abbreviated to $\bar{U}$ or $(U)^{-}$for $U \in T_{u \cdot v}(P \cdot Q)$. Let ${ }^{P \cdot Q \nabla}$ be the Riemannian connection of $\begin{gathered}P \cdot Q \\ \omega \diamond \eta\end{gathered}$ Proposition 1.3 by $\rho: T(P \cdot Q) \rightarrow \operatorname{Ker} d \pi_{P \cdot Q} ; X \mapsto X^{\top}, \rho^{\perp}: T(P \cdot Q) \rightarrow \operatorname{Ker} \omega \diamond \eta$; $X \mapsto X^{\perp} . \quad{ }^{P \cdot Q} A=\left[(X, Y) \mapsto\left({ }^{P \cdot Q} \nabla_{X^{\perp}} Y^{\top}\right)^{\perp}+\left({ }^{P \cdot Q} \nabla_{X} Y^{\perp}\right)^{\top}\right]$ is called the O'Neill's tensor

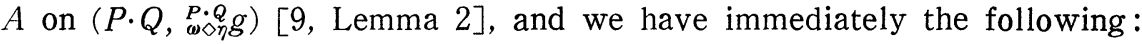

Proposition 2.2. For any $u \in P, v \in Q, X, Y \in T_{u \cdot v}(P \cdot Q)$,

$$
\left((\omega \diamond \eta){ }^{P \cdot Q} \mathcal{A}\right)_{u \cdot v}(X, Y)=\left[(u, v),-\frac{1}{2}\left(\left(p_{P}^{*}\right)^{\omega} \Omega+\left(p_{Q}^{*}\right)^{\eta} \Omega\right)(\bar{X}, \bar{Y})\right] .
$$


Let ${ }^{\omega \diamond \eta} \nabla$ be the covariant differentiation on $P \cdot Q$ with respect to $\omega \star \eta$ in $(P \times Q) \times \operatorname{Ad}_{G} \mathrm{~g}$ and ${ }_{P}^{\omega} * Q_{Q} \nabla$ be the induced differentiation on $P \cdot Q$ with respect to ${ }^{\omega \star \eta} \nabla$ and ${ }^{P \cdot Q} \nabla$ in $T^{*}(P \cdot Q) \otimes(P \times Q) \times{ }_{\mathrm{Ad}_{G}} g$. The Riemannian connection of ${ }_{\omega}^{P} g$, ${ }_{\eta}^{Q} g$ or ${ }^{P \times Q} g$ is denoted by ${ }^{P} \nabla,{ }^{Q} \nabla$ or ${ }^{P \times Q} \nabla$, respectively.

COROLlary 2.3. For any $u \in P, v \in Q, X, Y, Z \in T_{u \cdot v}(P \cdot Q)$,

$\left.{ }_{P}^{\omega} \star_{Q}^{\prime \prime} \nabla\left((\omega \diamond \eta){ }^{P \cdot Q} \mathcal{A}\right)\right)_{u \cdot v}(X, Y, Z)=\left[(u, v),-\frac{1}{2}\left(\left(p_{P}^{*}\right)^{P} \nabla^{\omega} \Omega+\left(p_{Q}^{*}\right)^{Q} \nabla^{\eta} \Omega\right)(\bar{X}, \bar{Y}, \bar{Z})\right]$.

Proposition 1.4 implies the following lemma:

LEMMA 2.4.

$$
\begin{aligned}
& \left.{ }^{\left({ }^{\star} \eta \eta\right.} d(\omega \diamond \eta)\right)_{u \cdot v}(X, Y)=0 \\
& \quad \text { for all } u \in P, v \in Q, X \in T_{u \cdot v}(P \cdot Q), Y \in \operatorname{Ker}\left(d \pi_{P \cdot Q}\right)_{u \cdot v} .
\end{aligned}
$$

LEMMA 2.5. For any $X, Y \in \mathscr{X}(P \cdot Q)$,

$$
(\omega \diamond \eta)\left({ }^{P \cdot Q} \nabla_{X^{\perp}} Y^{\top}\right)={ }^{\omega \star \eta} \nabla_{X^{\perp}}\left((\omega \diamond \eta)\left(Y^{\top}\right)\right) .
$$

Proof. Since each fiber of $P \cdot Q$ is totally geodesic and the decomposition in Proposition 1.3 is orthogonal with respect to the lifted metric,

$$
(\omega \diamond \eta)\left({ }^{P \cdot Q} \nabla_{Y^{\top}} X^{\perp}\right)=(\omega \diamond \eta)\left(\left({ }^{P \cdot Q} \nabla_{Y^{\top}} X^{\perp}\right)^{\perp}\right)=0
$$

therefore $(\omega \diamond \eta)\left(P^{P} \cdot \nabla_{X^{\perp}} Y^{\top}\right)=(\omega \diamond \eta)\left(\left[X^{\perp}, Y^{\top}\right]\right)$. By Lemma 2.4,

$$
\begin{aligned}
0 & =\left({ }^{\omega \star \eta} d(\omega \diamond \eta)\right)\left(X^{\perp}, Y^{\top}\right) \\
& =\frac{1}{2}\left\{{ }^{\omega \star \eta} \nabla_{X}\left((\omega \diamond \eta)\left(Y^{\top}\right)\right)-{ }^{\omega \star \eta} \nabla_{Y^{\top}}\left((\omega \diamond \eta)\left(X^{\perp}\right)\right)-(\omega \diamond \eta)\left(\left[X^{\perp}, Y^{\top}\right]\right)\right\} \\
& =\frac{1}{2}\left\{{ }^{\omega \star \eta} \nabla_{X^{\perp}}\left((\omega \diamond \eta)\left(Y^{\top}\right)\right)-(\omega \diamond \eta)\left(\left[X^{\perp}, Y^{\top}\right]\right)\right\} .
\end{aligned}
$$

Hence $(\omega \diamond \eta)\left(P^{P \cdot Q} \nabla_{X^{\perp}} Y^{\top}\right)={ }^{\omega \star \eta} \nabla_{X \perp}\left((\omega \diamond \eta)\left(Y^{\top}\right)\right)$.

LEMMA 2.6. For any $u \in P, v \in Q, X, Y, Z \in \operatorname{Ker}(\omega \diamond \eta)_{u \cdot v}$,

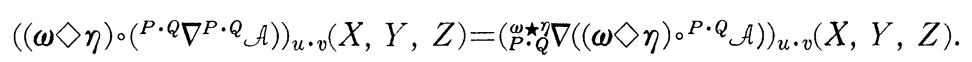

From Corollary 2.3 and Lemma 2.6, we have

Proposition 2.7. For any $u \in P, v \in Q, X, Y, Z \in \operatorname{Ker}(\omega \diamond \eta)_{u v}$,

$$
\left((\omega \diamond \eta){ }^{P}{ }^{P} Q \nabla^{P \cdot Q} \mathcal{A}\right)_{u \cdot v}(X, Y, Z)=\left[(u, v),-\frac{1}{2}\left(\left(p_{P}^{*}\right)^{P} \nabla^{\omega} \Omega+\left(p_{Q}^{*}\right)^{Q} \nabla^{\eta} \Omega\right)(\bar{X}, \bar{Y}, \bar{Z})\right] .
$$

$\omega($ resp. $\eta)$ is called a parallel connectıon if ${ }^{P} \nabla^{\omega} \Omega(\operatorname{Ker} \omega, \operatorname{Ker} \omega, \operatorname{Ker} \omega) \equiv 0$ 
(resp. ${ }^{Q} \nabla^{\eta} \Omega(\operatorname{Ker} \eta$, Ker $\eta$, Ker $\left.\eta) \equiv 0\right)$. For instance, in the canonical fibration over a Riemannian symmetric space, the canonical invariant connection with respect to its symmetric pair is a parallel connection [4].

COROLLARY 2.8. $\omega$ and $\eta$ are both parallel if and only if

$$
\left((\omega \diamond \eta)^{\circ P Q Q} \nabla^{P \cdot Q} \mathcal{A}\right)_{u \cdot v}(X, Y, Z)=0 \text { for all } u \in P, v \in Q, X, Y, Z \in \operatorname{Ker}(\omega \diamond \eta)_{u \cdot v} \text {. }
$$

Let ${ }^{\omega} D$ (resp. ${ }^{\eta} D$ ) be the exterior covariant differentiation on $P$ (resp. $Q$ ) with respect to $\omega$ (resp. $\eta$ ) and ${ }^{\omega} D^{*}$ (resp. ${ }^{\eta} D^{*}$ ) be the exterior covariant codifferentiation on $P$ (resp. $Q$ ) of ${ }^{\omega} D$ (resp. ${ }^{\eta} D$ ).

Proposition 2.9. For any $u \in P, v \in Q, Z \in \operatorname{Ker}(\omega \diamond \eta)_{u} \cdot v$,

$$
\operatorname{Trace}\left((\omega \diamond \eta)^{\circ} P \cdot Q \nabla^{P \cdot Q} \mathcal{A}\right)_{u \cdot v}\left(\rho^{\perp}, \rho^{\perp}, Z\right)=\left[(u, v), \frac{1}{2}\left(\left(p_{P}^{*}\right)^{\omega} D^{* \omega} \Omega+\left(p_{Q}^{*}\right)^{\eta} D^{* \eta} \Omega\right)(\bar{Z})\right] \text {. }
$$

$\omega$ (resp. $\eta$ ) is called a Yang-Mills connection if $\omega$ (resp. $\eta$ ) satisfies the Yang-Mills equation: ${ }^{\omega} D^{* \omega} \Omega \equiv 0$ (resp. ${ }^{\eta} D^{* \eta} \Omega \equiv 0$ ) (c. f. [2]). For instance, parallel connections are Yang-Mills connections.

COROLLARY 2.10. $\omega$ and $\eta$ are both Yang-Mills if and only if

Trace $\left((\omega \diamond \eta)_{0}{ }^{P \cdot Q} \nabla^{P \cdot Q} \mathcal{A}\right)_{u \cdot v}\left(\rho^{\perp}, \rho^{\perp}, Z\right)=0$ for all $u \in P, v \in Q, Z \in \operatorname{Ker}(\omega \diamond \eta)_{u \cdot v}$.

Notice that we can describe a pair of the Yang-Mills equations for $\omega$ and $\eta$ in terms of the Ricci curvature tensor ${ }^{P \cdot Q}$ Ric of $(P \cdot Q, \stackrel{P \cdot Q}{\omega \diamond \eta} g)$.

Proposition 2.11. For any $u \in P, v \in Q, Z \in \operatorname{Ker}(\omega \diamond \eta)_{u \cdot v}$,

$$
\left((\omega \diamond \eta)_{\circ}{ }^{P \cdot Q} R i c\right)_{u \cdot v}(Z)=\left[(u, v), \frac{1}{2}\left(\left(p_{P}^{*}\right)^{\omega} D^{* \omega} \Omega+\left(p_{Q}^{*}\right)^{\eta} D^{* \eta} \Omega\right)(\bar{Z})\right] .
$$

A pair of the Yang-Mills equations for $\omega$ and $\eta$ is a necessary condition for the Einstein equation with respect to $(P \cdot Q, \underset{\omega \diamond \eta}{P \cdot Q} g)$ as an analog to the relation between the Yang-Mills equation for $\omega$ and the Einstein with respect to $(P, \underset{P}{\omega} g)$ [12, Corollary 2.19].

COROLLARY 2.12 .

(i) $\omega$ and $\eta$ are both Yang-Mills if and only if

$$
{ }^{P \cdot Q} \operatorname{Ric}\left(\operatorname{Ker} d \pi_{P \cdot Q}, \operatorname{Ker} \omega \diamond \eta\right)=0 .
$$

(ii) If $\left(P \cdot Q, \begin{array}{c}P \cdot Q \\ \omega \diamond \eta\end{array}\right)$ is an Einstern space, then $\omega$ and $\eta$ are both Yang-Mills.

In the case of $N=M, Q=P^{-1}$ and $\eta=\omega^{-1}$, the inversion $i:(P, \underset{P}{\boldsymbol{\omega}} g) \rightarrow\left(P^{-1}\right.$, $P_{P}^{\omega-1} g$ ) is an isometry. Therefore we have immediately the followings:

COROLLARY 2.13. $\omega$ is parallel if and only if 


$$
\begin{aligned}
& \left(\left(\omega \diamond \omega^{-1}\right)_{o^{P} \cdot P^{-1}} \nabla^{P \cdot P^{-1}} \mathcal{A}\right)_{u \cdot v^{-1}}(X, Y, Z)=0 \\
& \quad \text { for all } u, v \in P, X, Y, Z \in \operatorname{Ker}\left(\omega \diamond \omega^{-1}\right)_{u \cdot v-1} .
\end{aligned}
$$

COROLlary 2.14. $\omega$ is Yang-Mills if and only if

$$
\begin{gathered}
\text { Trace }\left(\left(\omega \diamond \omega^{-1}\right)_{{ }^{P} \cdot P^{-1}} \nabla^{P \cdot P^{-1}} \mathcal{A}\right)_{u \cdot v^{-1}}\left(\rho^{\perp}, \rho^{\perp}, Z\right)=0 \\
\text { for all } u, v \in P, Z \in \operatorname{Ker}\left(\omega \diamond \omega^{-1}\right)_{u \cdot v^{-1}} .
\end{gathered}
$$

COROLLARY 2.15 .

(i) $\omega$ is Yang-Mills if and only if ${ }^{P \cdot P^{-1}} \operatorname{R} \imath$ (Ker $\left.d \pi_{P \cdot P-1}, \operatorname{Ker} \omega \diamond \omega^{-1}\right)=0$.

(ii) If $\left(P \cdot P^{-1}, \begin{array}{c}P \cdot P^{-1} \\ \omega \diamond \omega^{-1}\end{array}\right)$ is an Einstein space, then $\omega$ is Yang-Mills.

The diagonal map $\Delta_{M}:\left(M, 2^{M} g\right) \rightarrow\left(M \times M,^{M \times M} g\right)$ is a totally geodesic isometric embedding so that Corollary 1.19 implies the following:

Proposition 2.16. $\varepsilon:\left(M, 2^{M} g\right) \rightarrow\left(P \cdot P^{-1}, \begin{array}{c}P \cdot P-1 \\ \omega \diamond \omega^{-1}\end{array}\right)$ is a totally geodesıc isometric embedding for any connection form $\omega$ on $P$.

COROLLARY 2.17 .

(i) $\varepsilon^{* P \cdot P^{-1}} \mathcal{A}=0$, equivalently, for any $x \in M, X, Y \in T_{x} M, u \in \pi_{P}^{-1}(x)$,

$$
\left(\left(\boldsymbol{\omega} \diamond \omega^{-1}\right){ }_{\circ}^{P \cdot P-1} \mathcal{A}\right)\left(\omega \diamond \omega^{-1} X^{(+)},{ }^{\omega \diamond \omega^{-1}} Y^{(+)}\right)=0 .
$$

(ii) For any $x \in M, X, Y \in T_{x} M, u \in \pi_{P}^{-1}(x)$,

$$
\left(\left(\omega \diamond \omega^{-1}\right)_{\circ} P \cdot P^{-1} \mathcal{A}\right)\left(\omega \diamond \omega^{-1} X^{(-)}, \omega \diamond \omega^{-1} Y^{(-)}\right)=0 .
$$

Under the identification $\varepsilon^{-1}\left(P \times P^{-1}\right) \cong P$, we observe the following:

Proposition 2.18. For any $x \in M, X, Y \in T_{x} M, u \in \pi_{P}^{-1}(x)$,

$$
\left(\left(\omega \diamond \omega^{-1}\right){ }^{P \cdot P-1} \mathcal{A}\right)\left({ }^{\omega \diamond \omega^{-1}} X^{(+)},{ }^{\omega \diamond \omega^{-1}} Y^{(-)}\right)=\left[u,-{ }^{\omega} \Omega_{u}\left({ }^{\omega} H_{x}^{u} X,{ }^{\omega} H_{x}^{u} Y\right)\right] .
$$

COROLlary 2.19. $\omega$ is flat if and only if

$$
\left(\left(\omega \diamond \omega^{-1}\right){ }^{P \cdot P^{-1}} \mathcal{A}\right)\left(\omega^{\omega} \omega^{-1} X^{(+)}, \omega_{\diamond} \omega^{-1} Y^{(-)}\right)=0
$$

for all $x \in M, X, Y \in T_{x} M, u \in \pi_{P}^{-1}(x)$, equivalently,

$$
\left(\left(\boldsymbol{\omega} \diamond \boldsymbol{\omega}^{-1}\right)_{\mathrm{o}}^{P \cdot P-1} \mathcal{A}\right)\left((d \varepsilon)_{x} T_{x} M, \operatorname{Ker}\left(\boldsymbol{\omega} \diamond \boldsymbol{\omega}^{-1}\right)_{\varepsilon(x)}\right)=0, \quad \text { for all } x \in M \text {. }
$$

By using the fact that the inversion $\imath$ is an isometry, we get

Lemma 2.20. For any $x \in M, X, Y, Z \in T_{x} M, u \in \pi_{P}^{-1}(x)$,

$$
\begin{aligned}
& \left(\left(\omega \diamond \omega^{-1}\right){ }^{P \cdot P} P^{-1} \nabla^{P \cdot P^{-1}} \mathcal{A}\right)\left(\omega^{\omega \diamond \omega^{-1}} X^{(+)},{ }^{\omega \diamond \omega^{-1}} Y^{(+)},{ }^{\omega \diamond \omega^{-1}} Z^{(+)}\right)=0, \\
& \left(\left(\omega \diamond \omega^{-1}\right){ }^{P \cdot P^{-1}} \nabla^{P \cdot P-1} \mathcal{A}\right)\left({ }^{\left(\omega \diamond \omega^{-1}\right.} X^{(-)},{ }^{\omega \diamond \omega^{-1}} Y^{(-)}, \omega \diamond \omega^{-1} Z^{(+)}\right)=0,
\end{aligned}
$$




$$
\begin{aligned}
& \left(\left(\omega \diamond \omega^{-1}\right){ }_{\circ}^{P \cdot P-1} \nabla^{P \cdot P-1} \mathcal{A}\right)\left(\omega \diamond \omega^{-1} X^{(-)},{ }^{\omega \diamond \omega^{-1}} Y^{(+)}, \omega \diamond \omega^{-1} Z^{(-)}\right)=0, \\
& \left(\left(\boldsymbol{\omega} \diamond \omega^{-1}\right){ }_{\circ}^{P \cdot P-1} \nabla^{P \cdot P-1} \mathcal{A}\right)\left({ }^{\omega \diamond \omega^{-1}} X^{(+)},{ }^{\omega \diamond \omega^{-1}} Y^{(-)},{ }^{\omega \diamond \omega^{-1}} Z^{(-)}\right)=0, \\
& \left(\left(\omega \diamond \omega^{-1}\right){ }^{P \cdot P^{-1}} \nabla^{P \cdot P-1} \mathcal{A}\right)\left(\omega \diamond \omega^{-1} X^{(+)},{ }^{\omega \diamond \omega^{-1}} Y^{(+)}, \omega \diamond \omega^{-1} Z^{(-)}\right) \\
& =\left[u,-\left({ }^{P} \nabla^{\omega} \Omega\right)_{u}\left({ }^{\omega} H_{x}^{u} X,{ }^{\omega} H_{x}^{u} Y,{ }^{\omega} H_{x}^{u} Z\right)\right], \\
& \left(\left(\omega \diamond \omega^{-1}\right)_{\circ} P \cdot P^{-1} \nabla^{P \cdot P-1} \mathcal{A}\right)\left(\omega \diamond \omega^{-1} X^{(-)},{ }^{\omega \diamond \omega^{-1}} Y^{(-)}, \omega \diamond \omega^{-1} Z^{(-)}\right) \\
& =\left[u,-\left({ }^{P} \nabla^{\omega} \Omega\right)_{u}\left({ }^{\omega} H_{x}^{u} X,{ }^{\omega} H_{x}^{u} Y,{ }^{\omega} H_{x}^{u} Z\right)\right], \\
& \left(\left(\omega \diamond \omega^{-1}\right)_{\circ} P \cdot P^{-1} \nabla^{P \cdot P-1} \mathcal{A}\right)\left(\omega \diamond \omega^{-1} X^{(+)},{ }^{\omega \diamond \omega^{-1}} Y^{(-)}, \omega \diamond \omega^{-1} Z^{(+)}\right) \\
& =\left[u,\left({ }^{P} \nabla^{\omega} \Omega\right)_{u}\left({ }^{\omega} H_{x}^{u} X,{ }^{\omega} H_{x}^{u} Y,{ }^{\omega} H_{x}^{u} Z\right)\right], \\
& \left(\left(\omega \diamond \omega^{-1}\right)_{\circ} P \cdot P^{-1} \nabla^{P \cdot P^{-1}} \mathcal{A}\right)\left(\omega \diamond \omega^{-1} X^{(-)},{ }^{\omega \diamond \omega^{-1}} Y^{(+)}, \omega \diamond \omega^{-1} Z^{(+)}\right) \\
& =\left[u,-\left({ }^{P} \nabla^{\omega} \Omega\right)_{u}\left({ }^{\omega} H_{x}^{u} X,{ }^{\omega} H_{x}^{u} Y,{ }^{\omega} H_{x}^{u} Z\right)\right] .
\end{aligned}
$$

Proposition 1.16 and the above lemma imply the followings:

Proposition 2.21. For any $x \in M, u \in \pi_{P}^{-1}(x)$, the following three conditions are equivalent;

(i ) $\left(\left(\boldsymbol{\omega} \diamond \omega^{-1}\right)_{0} P^{\cdot P^{-1}} \nabla^{P \cdot P^{-1}} \mathcal{A}\right)\left((d \varepsilon)_{x} T_{x} M,(d \varepsilon)_{x} T_{x} M, \operatorname{Ker}\left(\omega \diamond \omega^{-1}\right)_{\varepsilon(x)}\right)=0$,

(ii ) $\left(\left(\boldsymbol{\omega} \diamond \boldsymbol{\omega}^{-1}\right)_{\circ}{ }^{P \cdot P-1} \nabla^{P \cdot P^{-1}} \mathcal{A}\right)\left(\operatorname{Ker}\left(\boldsymbol{\omega} \diamond \boldsymbol{\omega}^{-1}\right)_{\varepsilon(x)}, \operatorname{Ker}\left(\boldsymbol{\omega} \diamond \boldsymbol{\omega}^{-1}\right)_{\varepsilon(x)}, \operatorname{Ker}\left(\boldsymbol{\omega} \diamond \boldsymbol{\omega}^{-1}\right)_{\varepsilon(x)}\right)$ $=0$,

(iii) $\left({ }^{P} \nabla^{\omega} \Omega\right)_{u}\left(\operatorname{Ker} \omega_{u}, \operatorname{Ker} \omega_{u}, \operatorname{Ker} \omega_{u}\right)=0$.

COROLLARY 2.22. $\omega$ is parallel if and only if

$$
\left(\left(\boldsymbol{\omega} \diamond \boldsymbol{\omega}^{-1}\right)_{\circ}{ }^{P \cdot P-1} \nabla^{P \cdot P-1} \mathcal{A}\right)\left((d \varepsilon)_{x} T_{x} M,(d \varepsilon)_{x} T_{x} M, \operatorname{Ker}\left(\boldsymbol{\omega} \diamond \boldsymbol{\omega}^{-1}\right)_{\varepsilon(x)}\right)=0
$$

for all $x \in M$.

Let $\left\{E_{i}\right\}_{i=1}^{m}$ be an orthonormal basis for $\left(T_{x} M, 2^{M} g\right)$, then $\left\{{ }^{\omega \diamond \omega^{-1}} E_{\imath}^{(+)}\right.$, $\left.\omega \diamond \omega^{-1} E_{\imath}^{(-)}\right\}_{\imath=1}^{m}$ is an orthonormal basis for $\left(\operatorname{Ker}\left(\omega \diamond \omega^{-1}\right)_{\varepsilon(x)}, \begin{array}{c}P \cdot P-1 \\ \omega \diamond \omega^{-1}\end{array}\right.$ $\operatorname{dim} M$. The following lemma is obtained from Lemma 2.20:

LEMMA 2.23. For any $x \in M, Z \in T_{x} M, u \in \pi_{P}^{-1}(x)$,

$$
\begin{aligned}
& \sum_{\imath=1}^{m}\left(\left(\omega \diamond \omega^{-1}\right){ }_{\circ}^{P \cdot P-1} \nabla^{P \cdot P^{-1}} \mathcal{A}\right)\left({ }^{\omega \diamond} \omega^{-1} E_{\imath}^{(+)},{ }^{\omega \diamond \omega^{-1}} E_{\imath}^{(+)},{ }^{\omega \diamond \omega^{-1}} Z^{(+)}\right)=0, \\
& \sum_{\imath=1}^{m}\left(\left(\omega \diamond \omega^{-1}\right){ }_{\circ} P \cdot P^{-1} \nabla^{P \cdot P^{-1}} \mathcal{A}\right)\left({ }^{\omega \diamond \omega^{-1}} E_{\imath}^{(-)},{ }^{\omega \diamond \omega^{-1}} E_{\imath}^{(-)}, \omega \diamond \omega^{-1} Z^{(+)}\right)=0,
\end{aligned}
$$




$$
\begin{aligned}
& \sum_{\imath=1}^{m}\left(\left(\omega \diamond \omega^{-1}\right){ }^{P \cdot P-1} \nabla^{P \cdot P-1} \mathcal{A}\right)\left({ }^{\omega \diamond \omega^{-1}} E_{\imath}^{(+)}, \omega \diamond \omega^{-1} E_{\imath}^{(+)},{ }^{\omega \diamond \omega-1} Z^{(-)}\right) \\
& \quad=\left[u, \frac{1}{2}\left({ }^{\omega} D^{* \omega} \Omega\right)_{u}\left({ }^{\omega} H_{x}^{u} Z\right)\right], \\
& \sum_{\imath=1}^{m}\left(\left(\omega \diamond \omega^{-1}\right){ }^{P \cdot P-1} \nabla^{P \cdot P-1} \mathcal{A}\right)\left({ }^{\omega} \diamond \omega^{-1} E_{\imath}^{(-)},{ }^{\omega \diamond \omega^{-1}} E_{\imath}^{(-)},{ }^{\omega \diamond \omega-1} Z^{(-)}\right) \\
& \quad=\left[u, \frac{1}{2}\left({ }^{\omega} D^{* \omega} \Omega\right)_{u}\left({ }^{\omega} H_{x}^{u} Z\right)\right] .
\end{aligned}
$$

Proposition 1.9 and the above lemma imply the following:

Proposition 2.24. $\omega$ is Yang-Mills if and only if

$$
\begin{aligned}
& \sum_{\imath=1}^{m}\left(\left(\boldsymbol{\omega} \diamond \boldsymbol{\omega}^{-1}\right)_{{ }^{P} \cdot P^{-1}} \nabla^{P \cdot P-1} \mathcal{A}\right)\left((d \varepsilon)_{x} E_{\imath},(d \varepsilon)_{x} E_{\imath}, \operatorname{Ker}\left(\boldsymbol{\omega} \diamond \boldsymbol{\omega}^{-1}\right)_{\varepsilon(x)}\right)=0 \\
& \quad \text { for all } x \in M .
\end{aligned}
$$

\section{$\S 3$. Gauss Sections on the Joint Spaces}

Let $G_{r}(T(P \cdot Q)) \stackrel{\pi}{\rightarrow} P \cdot Q$ be the Grassmann bundle associated to $T(P \cdot Q)$ which typical fiber is the real Grassmann manifold of the $r$-dimensional planes, where $r=\operatorname{dim} G$. When we choose the Riemannian metric $\underset{\omega \bullet \ell}{P} g, G_{r}(T(P \cdot Q))$

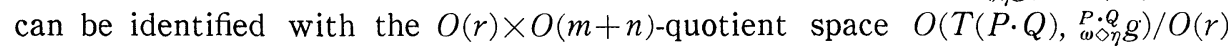
$\times O(m+n)$ of the orthonormal frame bundle $O(T(P \cdot Q), \underset{\omega \diamond \eta}{P} g)$ of $T(P \cdot Q)$ with respect to $\begin{gathered}P \cdot Q \\ \omega \diamond \eta\end{gathered}$, where $m=\operatorname{dim} M, n=\operatorname{dim} N$. The quotient map of $O\left(T(P \cdot Q), \begin{array}{c}P \cdot Q \\ \omega \diamond \eta\end{array}\right)$ onto $G_{r}(T(P \cdot Q))$ is denoted by $\zeta$, which is a right principal $O(r) \times O(m+n)$ fibration. Since the structure group of $\pi^{-1} T(P \cdot Q)$ is reduced to $O(r) \times O(m+n)$, the $O(r) \times O(m+n)$-submodule splitting $\boldsymbol{R}^{r+m+n}=\left(\boldsymbol{R}^{r}, 0_{m+n}\right) \oplus\left(0_{r}, \boldsymbol{R}^{m+n}\right)$ induces the vector subbundle splitting $\pi^{-1} T(P \cdot Q)=K \oplus K^{\perp}$ where

$$
\begin{aligned}
& K= {\left[O\left(T(P \cdot Q), \begin{array}{c}
P \cdot Q \\
\omega \diamond \eta
\end{array}\right) \stackrel{\zeta}{\longrightarrow} G_{r}(T(P \cdot Q))\right] \times{ }_{\sigma}\left(\boldsymbol{R}^{r}, 0_{m+n}\right), } \\
& K^{\perp}=\left[O\left(T(P \cdot Q), \begin{array}{c}
P \cdot Q \\
\omega \diamond \eta
\end{array}\right) \stackrel{\zeta}{\longrightarrow} G_{r}(T(P \cdot Q))\right] \times{ }_{\sigma}\left(0_{r}, \boldsymbol{R}^{m+n}\right) \text { and } \\
& \sigma: O(r) \times O(m+n) \subset G L\left(\boldsymbol{R}^{r+m+n}\right) \text { is the natural linear } \\
& \quad \text { representation of } O(r) \times O(m+n) .
\end{aligned}
$$

On the other hand, the Riemannian connection ${ }^{P \cdot Q} \nabla$ of $\underset{\omega}{P \bullet \eta} Q Q$ induces the splitting $T\left(G_{r}(T(P \cdot Q))\right)=\operatorname{Ker} d \pi \oplus(\operatorname{Ker} d \pi)^{\perp}$ so that the differential $d \gamma$ of $\gamma=\gamma_{P \cdot Q}$ splits into $(d \gamma)^{V}$ and $(d \gamma)^{H}$ where the former is the vertical differential of $\gamma$ with respect to ${ }^{P \cdot Q} \nabla[11],[12] . \quad \gamma$ is called a horizontal section with respect to ${ }^{P \cdot Q} \nabla$ if $(d \gamma)^{V} \equiv 0$. The vertıcal energy density of $\gamma$ is the $C^{\infty}$-function $\boldsymbol{e}^{V}(\gamma): P \cdot Q \rightarrow \boldsymbol{R}$ defined by $\boldsymbol{e}^{V}(\gamma)(u \cdot v)=\left\|(d \gamma)^{V}\right\|_{u \cdot v}^{2}(u \in P, v \in Q)$.

Let $\mathcal{K}$ be the vector subbundle $\left\{\left(\kappa,-\kappa^{\dagger}\right) \mid \kappa \in \operatorname{Hom}\left(K, K^{\perp}\right)\right\}$ of $\operatorname{Hom}\left(K, K^{\perp}\right) \oplus$ $\operatorname{Hom}\left(K^{\perp}, K\right)$ where $\kappa^{\dagger}$ is the adjoint of $\kappa$. C. M. Wood has introduced in [11] an 
isomorphism $\boldsymbol{l}=\left(\kappa,-\kappa^{\dagger}\right): \operatorname{Ker} d \pi \cong \mathcal{K} ;(d \zeta)_{E} A_{E}^{*} \mapsto E \circ A_{\mathfrak{t}} \circ E^{-1}(E \in O(T(P \cdot Q), \underset{\omega}{P \cdot Q} g)$, $A \in \mathfrak{v}(r+m+n))$ where $A_{\mathfrak{t}}$ is the $\mathrm{f}$-component of $A$ and $\mathfrak{t}$ is the orthogonal complement of $\mathfrak{v}(r) \times \mathfrak{v}(m+n)$ in $\mathfrak{v}(r+m+n)$ with respect to the Killing-Cartan form of $O(r+m+n)$ which is denoted by $1 / 2\left\langle\langle,\rangle . \quad \kappa: \operatorname{Ker} d \pi \rightarrow \operatorname{Hom}\left(K, K^{\perp}\right)\right.$ and $\kappa^{\dagger}: \operatorname{Ker} d \pi \rightarrow \operatorname{Hom}\left(K^{\perp}, K\right)$ are vector bundle isomorphisms. The metric is taken to be that derived from $1 / 2\langle$,$\rangle under the O(r) \times O(m+n)$-quotient map $O(r+m+n) \rightarrow G_{r}\left(\boldsymbol{R}^{r+m+n}\right)$. Thus $\boldsymbol{I}$ is 2-homothetic and $\kappa^{\dagger}$ is an isometry [12]. Note that $\gamma^{-1} K \cong \operatorname{Ker} d \pi_{P \cdot Q}, \gamma^{-1} K^{\perp} \cong \operatorname{Ker} \omega \diamond \eta$,

$$
\begin{aligned}
& \gamma^{-1} \operatorname{Hom}\left(K, K^{\perp}\right) \cong \operatorname{Hom}\left(\operatorname{Ker} d \pi_{P \cdot Q}, \operatorname{Ker} \omega \diamond \eta\right) \text { and } \\
& \gamma^{-1} \operatorname{Hom}\left(K^{\perp}, K\right) \cong \operatorname{Hom}\left(\operatorname{Ker} \omega \diamond \eta, \operatorname{Ker} d \pi_{P \cdot Q}\right) .
\end{aligned}
$$

We denote also the induced vector bundle isomorphisms via $\gamma$ by $I: \gamma^{-1} \operatorname{Ker} d \pi$ $\rightarrow \gamma^{-1} \mathcal{K}, \quad \kappa: \gamma^{-1} \operatorname{Ker} d \pi \rightarrow \operatorname{Hom}\left(\operatorname{Ker} d \pi_{P \cdot Q}, \operatorname{Ker} \omega \diamond \eta\right) \quad$ and $\quad \kappa^{\dagger}: \gamma^{-1} \operatorname{Ker} d \pi \rightarrow$ Hom $\left(\operatorname{Ker} \omega \diamond \eta, \operatorname{Ker} d \pi_{P \cdot Q}\right)$ for convenience of notation. $(d \gamma)^{V}$ is evaluated in $\operatorname{Hom}\left(\operatorname{Ker} \omega \diamond \eta, \operatorname{Ker} d \pi_{P \cdot Q}\right)$ as the O'Neill's tensor ${ }^{P \cdot Q} \mathcal{A}$ :

PROPOSITION 3.1.

(i) $\boldsymbol{I}\left((d \gamma)^{V} Y\right)=^{P \cdot Q} \mathcal{A}_{Y}=\left[W \mapsto^{P \cdot Q} \mathcal{A}(Y, W)\right]$, for any $u \in P, v \in Q, Y \in T_{u \cdot v}(P \cdot Q)$.

(ii) $2 \boldsymbol{e}^{V}(\gamma)(u \cdot v)=\left\|^{P \cdot Q} \mathcal{A}\right\|_{u}^{2} \cdot v$, for any $u \in P, v \in Q$.

Proof. (i) By the Gauss's and the Weingarten's formulas,

$$
\left({ }^{P \cdot Q} \nabla_{Y} W^{\top}\right)^{\perp}=\left({ }^{P \cdot Q} \nabla_{Y^{\perp}} W^{\top}\right)^{\perp}, \quad\left({ }^{P \cdot Q} \nabla_{Y} W^{\perp}\right)^{\top}=\left({ }^{P \cdot Q} \nabla_{Y^{\perp}} W^{\perp}\right)^{\top}
$$

since each fiber is totally geodesic. From [12, Corollary 1.9],

$$
\begin{aligned}
& \boldsymbol{I}\left((d \gamma)^{V} Y\right)=\left[W \mapsto\left({ }^{P \cdot Q} \nabla_{Y} W^{\perp}\right)^{\top}+\left({ }^{P \cdot Q} \nabla_{Y} W^{\top}\right)^{\perp}\right] \\
& =\left[W \mapsto\left({ }^{P \cdot Q} \nabla_{Y^{\perp}} W^{\perp}\right)^{\top}+\left({ }^{P \cdot Q} \nabla_{Y} W^{\top}\right)^{\perp}\right]=\left[W \mapsto{ }^{P \cdot Q} \mathcal{A}(Y, W)\right] .
\end{aligned}
$$

(ii) It follows from (i).

COROLLARY 3.2 .

(i) $\kappa^{\dagger}\left((d \gamma)^{V} Y\right)=\rho^{\circ} P \cdot Q A_{Y \perp} \circ \rho^{\perp}$, for any $u \in P, v \in Q, Y \in T_{u \cdot v}(P \cdot Q)$.

(ii) $\boldsymbol{e}^{V}(\gamma)(u \cdot v)=\left\|\rho^{\circ} P \cdot Q \mathcal{A}_{\rho^{\perp} \circ} \rho^{\perp}\right\|_{u \cdot v}^{2}$, for any $u \in P, v \in Q$.

PROPOSITION 3.3.

(i) For any $u \in P, v \in Q, Y \in T_{u \cdot v}(P \cdot Q), W \in \operatorname{Ker}(\omega \diamond \eta)_{u \cdot v}$,

$$
(\boldsymbol{\omega} \diamond \eta)\left(\kappa^{\dagger}\left((d \gamma)^{V} Y\right) W\right)=\left[(u, v),-\frac{1}{2}\left(\left(p_{P}^{*}\right)^{\omega} \Omega+\left(p_{Q}^{*}\right)^{\eta} \Omega\right)(\bar{Y}, \bar{W})\right] .
$$

(ii) $\boldsymbol{e}^{V}(\gamma)(u \cdot v)=1 / 2\left(\left\|^{\omega} \Omega\right\|_{u}^{2}+\left\|^{\eta} \Omega\right\|_{v}^{2}\right)$, for any $u \in P, v \in Q$.

Proof. (i) It follows from Proposition 2.2 and Corollary 3.2. (ii) Let $\left\{{ }^{M} E_{\imath}\right\}_{\imath=1}^{m},\left\{{ }^{N} E_{j}\right\}_{j=1}^{n}$ be orthonormal bases for $\left(T_{x} M,{ }^{M} g\right),\left(T_{y} N,{ }^{N} g\right)$, respectively, 
we set

$$
{ }^{P \cdot Q} E_{\imath}=\left(d_{\jmath_{P \cdot Q}}\right)_{(u, v)}\left({ }^{\omega} H_{x}^{u M} E_{\imath}, 0_{v}\right), \quad{ }^{P \cdot Q} E_{m+\jmath}=\left(d_{\jmath P \cdot Q}\right)_{(u, v)}\left(0_{u},{ }^{\eta} H_{y}^{v N} E_{\jmath}\right) .
$$

$\left\{{ }^{P \cdot Q} E_{\alpha}\right\}_{\alpha=1}^{m+n}$ is an orthonormal basis for $\left(\operatorname{Ker}(\omega \diamond \eta)_{u \cdot v},{ }^{P \cdot Q} g\right) . \quad(d \gamma)_{u \cdot v}^{V} \operatorname{Ker}\left(d \pi_{P \cdot Q}\right)_{u \cdot v}$ $=0$ so that

$$
\begin{aligned}
& \boldsymbol{e}^{V}(\gamma)(u \cdot v)=\sum_{\alpha=1}^{m+n}\left\|(d \gamma)^{V P \cdot Q} E_{\alpha}\right\|_{u \cdot v}^{2}=\sum_{\alpha=1}^{m+n}\left\|\kappa^{\dagger}\left((d \gamma)^{V P \cdot Q} E_{\alpha}\right)\right\|_{u \cdot v}^{2} \\
& =\sum_{\alpha, \beta=1}^{m+n}\left\|\kappa^{\dagger}\left((d \gamma)^{V P \cdot Q} E_{\alpha}\right)^{P \cdot Q} E_{\beta}\right\|_{u \cdot v}^{2}=2 \sum_{\alpha, \beta=1}^{m+n}\left\|(\omega \diamond \eta)\left(\kappa^{\dagger}\left((d \gamma)^{V P \cdot Q} E_{\alpha}\right)^{P \cdot Q} E_{\beta}\right)\right\|_{u \cdot v}^{2} \\
& =\frac{1}{2} \sum_{\alpha, \beta=1}^{m+n}\left\|\left(\left(p_{P}^{*}\right)^{\omega} \Omega+\left(p_{Q}^{*}\right)^{\eta} \Omega\right)\left(\left({ }^{P \cdot Q} E_{\alpha}\right)^{-},\left({ }^{P \cdot Q} E_{\beta}\right)^{-}\right)\right\|_{u \cdot v}^{2}=\frac{1}{2}\left(\|\omega \Omega\|_{u}^{2}+\|\eta \Omega\|_{v}^{2}\right) .
\end{aligned}
$$

THEOREM 3.4. $\omega$ and $\eta$ are both flat if and only if $\gamma_{P \cdot Q}$ is a horizontal section with respect to ${ }^{P \cdot Q} \nabla$.

COROLLARY 3.5. $\omega$ is flat if and only if $\gamma_{P \cdot P-1}$ is a horızontal section with respect to ${ }^{P \cdot P-1} \nabla$.

PROPOSITION 3.6.

(i) For any $x \in M, u \in \pi_{P}^{-1}(x), X, Y \in T_{x} M$,

$$
\begin{aligned}
& \left(\omega \diamond \omega^{-1}\right)\left(\kappa^{\dagger}\left(\left(d \gamma_{P \cdot P-1}\right)^{V \omega \diamond \omega^{-1}} X^{(+)}\right)^{\omega \diamond \omega^{-1}} Y^{(+)}\right)=0, \\
& \left(\omega \diamond \omega^{-1}\right)\left(\kappa^{\dagger}\left(\left(d \gamma_{P \cdot P-1}\right)^{V \omega \diamond \omega^{-1}} X^{(+)}\right)^{\omega \diamond \omega^{-1}} Y^{(-)}\right)=\left[u,-{ }^{\omega} \Omega\left({ }^{\omega} H_{x}^{u} X,{ }^{\omega} H_{x}^{u} Y\right)\right], \\
& \left(\omega \diamond \omega^{-1}\right)\left(\kappa^{\dagger}\left(\left(d \gamma_{P \cdot P-1}\right)^{V \omega \diamond \omega-1} X^{(-)}\right)^{\omega \diamond \omega^{-1}} Y^{(+)}\right)=\left[u,{ }^{\omega} \Omega\left({ }^{\omega} H_{x}^{u} X,{ }^{\omega} H_{x}^{u} Y\right)\right], \\
& \left(\omega \diamond \omega^{-1}\right)\left(\kappa^{\dagger}\left(\left(d \gamma_{P \cdot P-1}\right)^{V \omega \diamond \omega^{-1}} X^{(-)}\right)^{\omega \diamond \omega^{-1}} Y^{(-)}\right)=0 .
\end{aligned}
$$

(ii) $\left\|\left(d \gamma_{P \cdot P-1}\right)^{V \omega \diamond \omega^{-1}} X^{(+)}\right\|_{\varepsilon(x)}^{2}=\left\|\left(d \gamma_{P \cdot P-1}\right)^{V \omega \diamond \omega^{-1}} X^{(-)}\right\|_{\varepsilon(x)}^{2}$ for any $x \in M, X \in$ $T_{x} M$.

(iii) $\boldsymbol{e}^{V}\left(\gamma_{P \cdot P-1}\right)(\varepsilon(x))=2 \sum_{m}^{l=1}\left\|\left(d \gamma_{P \cdot P-1}\right)^{V \omega \diamond \omega^{-1}} E_{\imath}^{(+)}\right\|_{\varepsilon(x)}^{2}=\left\|{ }^{\omega} \Omega\right\|_{u}^{2}$ for anv $x \in M$, $u \in \pi_{P}^{-1}(x)$.

Proof. (i) It follows from Corollary 2.17. (ii) From (i), we get

$$
\begin{aligned}
& \left\|\left(d \gamma_{P \cdot P-1}\right)^{V \omega \diamond \omega^{-1}} X^{(+)}\right\|_{\varepsilon(x)}^{2}=\sum_{\imath=1}^{m}\left\|\kappa^{\dagger}\left(\left(d \gamma_{P \cdot P-1}\right)^{V \omega \diamond \omega^{-1}} X^{(+)}\right)^{\omega \diamond \omega \omega^{-1}} E_{\imath}^{(-)}\right\|_{\varepsilon(x)}^{2} \\
& =\frac{1}{2} \sum_{\imath=1}^{m}\left\|-{ }^{\omega} \Omega\left({ }^{\omega} H_{x}^{u} X,{ }^{\omega} H_{x}^{u} E_{\imath}\right)\right\|_{\varepsilon(x)}^{2}=\frac{1}{2} \sum_{\imath=1}^{m}\left\|\omega \Omega\left({ }^{\omega} H_{x}^{u} X,{ }^{\omega} H_{x}^{u} E_{\imath}\right)\right\|_{\varepsilon(x)}^{2} \\
& =\sum_{\imath=1}^{m}\left\|\kappa^{\dagger}\left(\left(d \gamma_{P \cdot P-1}\right)^{V \omega \diamond \omega \omega^{-1}} X^{(-)}\right)^{\omega \diamond \omega^{-1}} E_{\imath}^{(+)}\right\|_{\varepsilon(x)}^{2}=\left\|\left(d \gamma_{P \cdot P-1}\right)^{V \omega \diamond \omega-1} X^{(-)}\right\|_{\varepsilon(x)}^{2} .
\end{aligned}
$$

(iii) It follows from (ii). 
Let $\nabla^{V}$ be the induced connection in $\operatorname{Ker} d \pi$ from ${ }^{P \cdot Q} \nabla$ and ${ }^{\pi}(P \cdot Q \nabla)$ be the induced connection in $\pi^{-1} T(P \cdot Q)$ from ${ }^{P \cdot Q} \nabla$ and ${ }^{K} \nabla$ be the induced connection in $\mathcal{K}$ from ${ }^{\pi}\left({ }^{P \cdot Q} \nabla\right)$. Notice that $\boldsymbol{I}:\left(\operatorname{Ker} d \pi, \nabla^{V}\right) \rightarrow\left(\mathcal{K},{ }^{\mathcal{K}} \nabla\right)$ is not connectionpreserving but $I:\left(\gamma^{-1} \operatorname{Ker} d \pi, \nabla^{V}\right) \rightarrow\left(\gamma^{-1} \mathcal{K}, \gamma^{-1} \mathcal{K} \nabla\right)$ is connection-preserving, where ${ }^{r} \nabla^{V}$ (resp. $\left.{ }^{\gamma^{-1} K} \nabla\right)$ is the induced connection via $\gamma$ in $\gamma^{-1} \operatorname{Ker} d \pi$ (resp. $\gamma^{-1} \mathcal{K}$ ) from $\nabla^{V}\left(\right.$ resp. $\left.{ }^{\kappa} \nabla\right)[12$, Theorem 1.5, Corollary 1.6].

Proposition 3.7. For any $X, Y \in \mathscr{X}(P \cdot Q)$,

(i) $\boldsymbol{I}\left({ }^{\gamma} \nabla_{X}^{V}\left((d \gamma)^{V} Y\right)\right)=r^{-1} \mathcal{K}^{\mathcal{R}} \nabla_{X}^{P} \cdot Q \mathcal{A}_{Y}$,

(ii) $\kappa^{\dagger}\left({ }^{(r} \nabla_{X}^{V}\left((d \gamma)^{V} Y^{\top}\right)\right)=\rho{ }^{\gamma^{-1} \mathcal{K}} \nabla_{X}^{P \cdot Q} \mathcal{A}_{Y} \circ \rho^{\perp}$.

LEMMA 3.8. For any $X, Y, W \in \mathscr{X}(P \cdot Q)$,

$$
\left(\rho \circ\left({ }^{P \cdot Q} \nabla_{X}^{P \cdot Q} \mathcal{A}\right)_{Y} \rho^{\perp}\right)(W)=\left(\rho^{\circ} \gamma^{-1} \nVdash \nabla_{X}^{P} \cdot Q \mathcal{A}_{Y} \circ \rho^{\perp}\right)(W)-{ }^{P \cdot Q} \mathcal{A}\left(\left(\left(^{P \cdot Q} \nabla_{X} Y\right)^{\perp}, W^{\perp}\right) .\right.
$$

Let ${ }^{r} \nabla^{V}(d \gamma)^{V}$ be the vertical second fundamental form of $\gamma$ with respect to ${ }^{P \cdot Q} \nabla$ (definition in $[11]$ ). $\gamma$ will be called a covariantly horizontal section with respect to ${ }^{P \cdot Q} \nabla$ if ${ }^{\gamma} \nabla^{V}(d \gamma)^{V} \equiv 0$.

Proposition 3.9. For any $u \in P, v \in Q, X, Y, W \in T_{u \cdot v}(P \cdot Q)$,

(i) $\kappa^{\dagger}\left(\nabla^{V}(d \gamma)^{V}(X, Y)\right)(W)=\left({ }^{P \cdot Q} \nabla^{P \cdot Q} \mathcal{A}\left(X, Y, W^{\perp}\right)\right)^{\top}$,

(ii) $\kappa^{\dagger}\left(\nabla^{V}(d \gamma)^{V}\left(X^{\top}, Y^{\top}\right)\right)(W)=0$.

Proof. (i) From Proposition 3.7 and Lemma 3.8,

$$
\begin{aligned}
& \kappa^{\dagger}\left(\nabla^{V}(d \gamma)^{V}(X, Y)\right)(W)=\kappa^{\dagger}\left(\gamma \nabla_{X}^{V}\left((d \gamma)^{V} Y\right)\right)(W)-\kappa^{\dagger}\left((d \gamma)^{V}\left({ }^{P \cdot Q} \nabla_{X} Y^{\top}\right)\right)(W) \\
& =\left(\rho^{\circ \gamma^{-1} \mathcal{K}} \nabla_{X}^{P \cdot Q} \mathcal{A}_{Y} \circ \rho^{\perp}\right)(W)-\left(\rho^{\circ}{ }^{P \bullet Q} \mathcal{A}_{P \cdot Q} \nabla_{X} Y^{\circ} \rho^{\perp}\right)(W) \\
& =\left(\rho \circ r^{-1}{ }^{K} \nabla_{X}^{P} \cdot Q \mathcal{A}_{Y} \circ \rho^{\perp}\right)(W)-\left({ }^{P \cdot Q} \mathcal{A}\left({ }^{P \cdot Q} \nabla_{X} Y, W^{\perp}\right)\right)^{\top} \\
& =\left(\rho \circ \gamma^{-1 / \kappa} \nabla_{X}^{P \cdot Q} \mathcal{A}_{Y} \circ \rho^{\perp}\right)(W)-{ }^{P \cdot Q} \mathcal{A}\left(\left({ }^{P \cdot Q} \nabla_{X} Y\right)^{\perp}, W^{\perp}\right) \\
& =\left(\rho \circ\left(P^{P \cdot Q} \nabla_{X}^{P} \cdot Q \mathcal{A}\right)_{Y} \circ \rho^{\perp}\right)(W) .
\end{aligned}
$$

(ii) It follows from (i) and [9, Lemma 4].

Notice that ${ }^{r} \nabla^{V}(d \gamma)^{V}\left(X^{\top}, Y^{\perp}\right)$ and ${ }^{r} \nabla^{V}(d \gamma)^{V}\left(X^{\perp}, Y^{\top}\right)$ de not vanish, in general.

Corollary 3.10. For any $u \in P, v \in Q, X, Y \in T_{u \cdot v}(P \cdot Q), W \in \operatorname{Ker}(\boldsymbol{\omega} \diamond \eta)_{u \cdot v}$,

$$
\begin{aligned}
& (\omega \diamond \eta)_{u \cdot v}\left(\kappa^{\dagger}\left(r \nabla^{V}(d \gamma)^{V}(X, Y)\right)(W)\right) \\
& \quad=\left[(u, v),-\frac{1}{2}\left(\left(p_{P}^{*}\right)^{P} \nabla^{\omega} \Omega+\left(p_{Q}^{*}\right)^{Q} \nabla^{\eta} \Omega\right)(\bar{X}, \bar{Y}, \bar{W})\right] .
\end{aligned}
$$

Proof. It follows from Propositions 2.7 and 3.9.

THEOREM 3.11 .

(i) $\omega$ and $\eta$ are both parallel of and only if 
${ }^{r} \nabla(d \gamma)^{V}\left(\operatorname{Ker}(\boldsymbol{\diamond} \diamond \eta)_{u \cdot v}, \operatorname{Ker}(\boldsymbol{\omega} \diamond \eta)_{u \cdot v}\right)=0$, for all $u \in P, v \in Q$.

(ii) If $\gamma_{P \cdot Q}$ is a covariantly horizontal section with respect to ${ }^{P \cdot Q} \nabla$, then $\omega$ and $\eta$ are both parallel.

COROLLARY 3.12 .

(i) $\omega$ is parallel if and only if

${ }^{\gamma} \cdot P^{-1} \nabla^{V}\left(d \gamma_{P \cdot P^{-1}}\right)^{V}\left(\operatorname{Ker}\left(\omega \diamond \omega^{-1}\right)_{u \cdot v-1}, \operatorname{Ker}\left(\omega \diamond \omega^{-1}\right)_{u \cdot v-1}\right)=0$ for all $u, v \in P$.

(ii) If $\gamma_{P \cdot P^{-1}}$ is a covariantly horizontal section with respect to ${ }^{P \cdot P-1} \nabla$, then $\omega$ is parallel.

Let $\tau^{V}(\gamma)=\operatorname{Trace}^{\gamma} \nabla^{V}(d \gamma)^{V}$ be the vertical tension field of $\gamma$ with respect to $P \cdot Q \nabla . \quad \gamma$ is called a harmonic section (or vertical harmonic map) with respect to $P \cdot Q \nabla$ if $\gamma$ satisfies the harmonic section equation: $\tau^{V}(\gamma) \equiv 0$.

Proposition 3.13. For any $u \in P, v \in Q, W \in \operatorname{Ker}(\omega \diamond \eta)_{u \cdot v}$,

$$
(\boldsymbol{\omega} \diamond \eta)_{u \cdot v}\left(\kappa^{\dagger}\left(\tau^{V}(\gamma)\right)(W)=\left[(u, v), \frac{1}{2}\left(\left(p_{P}^{*}\right)^{\omega} D^{* \omega} \Omega+\left(p_{Q}^{*}\right)^{\eta} D^{* \eta} \Omega\right)(\bar{W})\right)\right] .
$$

Proof. From Proposition 3.9 (ii),

$$
\begin{aligned}
\kappa^{\dagger}\left(\tau^{V}(\gamma)\right)(W) & =\operatorname{Trace} \kappa^{\dagger}\left(r \nabla^{V}(d \gamma)^{V}(\rho, \rho)\right)(W)+\operatorname{Trace} \kappa^{\dagger}\left(r \nabla^{V}(d \gamma)^{V}\left(\rho^{\perp}, \rho^{\perp}\right)\right)(W) \\
& =\operatorname{Trace} \kappa^{\dagger}\left(r \nabla^{V}(d \gamma)^{V}\left(\rho^{\perp}, \rho^{\perp}\right)\right)(W)
\end{aligned}
$$

so that

$$
\begin{aligned}
(\omega \diamond \eta)\left(\kappa^{\dagger}\left(\tau^{V}(\gamma)\right)(W)\right) & =\operatorname{Trace}\left[(u, v),-\frac{1}{2}\left(\left(p_{P}^{*}\right)^{P} \nabla^{\omega} \Omega+\left(p_{Q}^{*}\right)^{Q} \nabla^{\eta} \Omega\right)\left(\bar{\rho}^{\perp}, \bar{\rho}^{\perp}, \bar{W}\right)\right] \\
& =\left[(u, v), \frac{1}{2}\left(\left(p_{P}^{*}\right)^{\omega} D^{* \omega} \Omega+\left(p_{Q}^{*}\right)^{\eta} D^{* \eta} \Omega\right)(\bar{W})\right] .
\end{aligned}
$$

THEOREM 3.14. $\omega$ and $\eta$ are both Yang-Mills if and only if $\gamma_{P \cdot Q}$ is a harmonic section with respect to ${ }^{P \cdot Q} \nabla$.

COROLLARY 3.15. $\omega$ is Yang-Mills if and only if $\gamma_{P \cdot P-1}$ is a harmonic section with respect to ${ }^{P \cdot P-1} \nabla$.

\section{§4. Gauss Sections along Object Inclusion Map}

In this section, we prepare a general argument on the pull-back Gauss sections via a $C^{\infty}$-map and an application to the object inclusion map $\varepsilon: M \rightarrow P \cdot P^{-1}$.

Let $M$ and $L$ be $C^{\infty}$-manifolds and $\varphi: M \rightarrow L$ be a $C^{\infty}$-map. For a real $C^{\infty}$ vector bundle $\mathcal{E} \stackrel{\pi \varepsilon}{\rightarrow} L$ of rank $r+s+t(<+\infty)$, we denote the induced vector 
bundle from $\mathcal{E}$ via $\varphi$ by $\varphi^{-1} \mathcal{E} \stackrel{\varphi_{\pi \mathcal{E}}}{\longrightarrow} M$. The Grassmann bundle of the $r$-dimensional planes associated to $\mathcal{E}$ is denoted by $G_{r}(\mathcal{E}) \stackrel{{ }^{\pi} G_{r}(\mathcal{E})}{\longrightarrow} L$. Let $\mathscr{F}$ be a vector subbundle with rank $r$ of $\mathcal{E}$ and $\gamma_{\mathscr{T}}=\left[L \ni l \mapsto \mathscr{F}_{l} \subset \mathcal{E}_{l}\right]$ be the corresponding Gauss section into $G_{r}(\mathcal{E})$.

The induced fiber bundle $\varphi^{-1} G_{r}(\mathcal{E}) \stackrel{\varphi_{\pi G_{r}(\mathcal{E})}}{\longrightarrow} M$ is naturally identified with $G_{r}\left(\varphi^{-1} \mathcal{E}\right) \stackrel{\pi_{r}\left(\varphi^{-1} \mathcal{E}\right)}{\longrightarrow} M$. Let $\bar{\varphi}: G_{r}\left(\varphi^{-1} \mathcal{E}\right) \rightarrow G_{r}(\mathcal{E})$ be the induced fiber bundle homomorphism with $\pi_{\mathcal{E}} \circ \bar{\varphi}=\varphi^{\circ} \pi_{\mathcal{E}} \cdot \varphi^{-1} \mathscr{F}$ is naturally identified with a vector subbundle of $\varphi^{-1} \mathcal{E}$ so that $\gamma_{\varphi-1 \mathcal{F}}$ maps $M$ into $G_{r}\left(\varphi^{-1} \mathcal{E}\right)$ and $\bar{\varphi} \circ \gamma_{\varphi^{-1 \mathscr{F}}}=\gamma_{\mathscr{T}} \circ \varphi$.

Let $\left(\mathcal{E}, h_{\mathcal{E}}\right) \stackrel{\pi \mathcal{E}}{\rightarrow} L$ be a real $C^{\infty}$-vector bundle with a fiber metric $h_{\mathcal{E}}$ and $O\left(\mathcal{E}, h_{\mathcal{E}}\right) \stackrel{\pi^{O\left(\mathcal{E}, h_{\mathcal{E}}\right)} \longrightarrow}{\longrightarrow} L$ be the orthonormal frame bundle of $\left(\mathcal{E}, h_{\mathcal{E}}\right)$. For the induced vector bundle $\left(\varphi^{-1} \mathcal{E},{ }^{\varphi} h_{\mathcal{E}}\right) \stackrel{\varphi_{\pi \mathcal{E}}}{\longrightarrow} M$, the orthonormal frame bundle $O\left(\varphi^{-1} \mathcal{E},{ }^{\varphi} h_{\mathcal{E}}\right)$ is naturally identified with $\varphi^{-1} O\left(\mathcal{E}, h_{\mathcal{E}} \stackrel{\varphi_{\pi O(\mathcal{E}, h)}}{\longrightarrow} M\right.$. Let $\mathcal{E}_{\boldsymbol{\zeta}}$ (resp. $\varphi^{-1} \mathcal{E} \zeta$ ) be the quotient map $O\left(\mathcal{E}, h_{\mathcal{E}}\right) \rightarrow G_{r}(\mathcal{E})$ (resp. $O\left(\varphi^{-1} \mathcal{E},{ }^{\varphi} h_{\mathcal{E}}\right) \rightarrow G_{r}\left(\varphi^{-1} \mathcal{E}\right)$ ), which is a right principal $O(r) \times O(s+t)$-fibration. The pull-back vector bundle $\left(\pi_{G_{r}(\mathcal{E})}\right)^{-1} \mathcal{E} \rightarrow$ $G_{r}(\mathcal{E})\left(\right.$ resp. $\left.\left(\pi_{G_{r}(\varphi-1 \mathcal{E})}\right)^{-1}\left(\varphi^{-1} \mathcal{E}\right) \rightarrow G_{r}\left(\varphi^{-1} \mathcal{E}\right)\right)$ splits via $\pi_{G_{r}(\mathcal{E})}$ (resp. $\left.\pi_{G_{r}(\varphi-1 \mathcal{E})}\right)$ into ${ }^{\mathcal{E}} K \oplus^{\mathcal{E}} K^{\perp}$ (resp. ${ }^{\varphi^{-1} \mathcal{E}} K \oplus^{\varphi^{-1} \mathcal{E}} K^{\perp}$ ) where

$$
{ }^{\varepsilon} K=\left[O\left(\mathcal{E}, h_{\mathcal{E}}\right) \stackrel{\mathcal{E}_{\xi}}{\rightarrow} G_{r}(\mathcal{E})\right] \times_{\sigma}\left(\boldsymbol{R}^{r}, 0_{s+t}\right),{ }^{\mathcal{E}} K^{\perp}=\left[O\left(\mathcal{E}, h_{\mathcal{E}}\right) \stackrel{\mathcal{\varepsilon}_{\xi}}{\rightarrow} G_{r}(\mathcal{E})\right] \times_{\sigma}\left(0_{r}, \boldsymbol{R}^{s+t}\right)
$$

(resp. ${ }^{-1} \mathcal{E} K=\left[O\left(\varphi^{-1} \mathcal{E},{ }^{\varphi} h_{\mathcal{E}}\right) \stackrel{\varphi^{-1} \mathcal{E} \zeta}{\longrightarrow} G_{r}\left(\varphi^{-1} \mathcal{E}\right)\right] \times{ }_{\sigma}\left(\boldsymbol{R}^{r}, 0_{s+t}\right)$,

$$
\left.\varphi^{-1} \mathcal{C} K^{\perp}=\left[O\left(\varphi^{-1} \mathcal{E},{ }^{\varphi} h_{\mathcal{E}}\right) \stackrel{\varphi^{-1} \mathcal{E} \zeta}{\longrightarrow} G_{r}\left(\varphi^{-1} \mathcal{E}\right)\right] \times_{\sigma}\left(0_{r}, \boldsymbol{R}^{s+t}\right)\right)
$$

and $\sigma: O(r) \times O(s+t) \subset G L\left(\boldsymbol{R}^{r+s+t}\right)$ is the natural linear representation of $O(r)$ $\times O(s+t)$. There are natural vector bundle isomorphisms; $\bar{\varphi}^{-1}\left({ }^{\mathcal{E}} K\right) \cong{ }^{-1} \mathcal{E} K$, $\bar{\varphi}^{-1}\left({ }^{\mathcal{E}} K^{\perp}\right) \cong \varphi^{-1} \mathcal{E} K^{\perp}, \bar{\varphi}^{-1}$ End $\left(\left(\pi_{G_{r}(\mathcal{E})}\right)^{-1} \mathcal{E}\right) \cong \operatorname{End}\left(\left(\pi_{G_{r}(\varphi-1 \mathcal{E})}\right)^{-1}\left(\varphi^{-1} \mathcal{E}\right)\right)$.

As in $\S 3$, we set ${ }^{\mathcal{E}} \mathcal{K}=\left\{\left(\kappa,-\kappa^{\dagger}\right) \mid \kappa \in \operatorname{Hom}\left({ }^{\mathcal{E}} K,{ }^{\mathcal{E}} K^{\perp}\right)\right\}$,

$$
\varphi^{-1} \mathcal{E} \mathcal{K}=\left\{\left(\kappa,-\kappa^{\dagger}\right) \mid \kappa \in \operatorname{Hom}\left(\varphi^{-1} \mathcal{E} K, \varphi^{-1} \mathcal{E} K^{\perp}\right)\right\} .
$$

Under the natural identification $\bar{\varphi}^{-1}(\mathcal{E} \mathcal{K}) \cong \varphi^{-1} \mathcal{E} \mathcal{K}$, there is no confusion when we write $\bar{\varphi}: \varphi^{-1} \mathcal{E} \mathcal{K} \rightarrow{ }^{\mathcal{E}} \mathcal{K} .{ }^{\mathcal{E}} \boldsymbol{I}$ and ${ }^{\varphi^{-1} \mathcal{E}} \boldsymbol{I}$ are defined by

$$
\begin{gathered}
{ }^{\mathcal{I}} \boldsymbol{I}: \operatorname{Ker} d \pi_{G_{p}(\mathcal{E})} \cong{ }^{\mathcal{E}} \mathcal{K} ;\left(d^{\mathcal{E} \zeta}\right)_{E} A_{E}^{\#} \mapsto E \circ A_{\mathfrak{f}} \circ E^{-1}, \\
{ }^{-1} \mathcal{E} \boldsymbol{I}: \operatorname{Ker} d \pi_{G_{r}(\varphi-1 \mathcal{E})} \cong{ }^{-1} \mathcal{E} \mathcal{K} ;\left(d^{\varphi^{-1} \mathcal{E}} \zeta\right)_{\widetilde{E}} A_{\widetilde{E}}^{\#} \mapsto \tilde{E} \circ A_{\mathfrak{f}} \circ \tilde{E}^{-1}
\end{gathered}
$$

$\left(E \in O\left(\mathcal{E}, h_{\mathcal{E}}\right), \tilde{E} \in O\left(\varphi^{-1} \mathcal{E},{ }^{\varphi} h_{\mathcal{E}}\right), A \in \mathfrak{p}(r+s+t)\right)$, respectively, where $A_{\mathfrak{t}}$ is the $\mathfrak{f}$ component of $A$ and $\mathfrak{f}$ is the orthogonal complement of $\mathfrak{p}(r) \times \mathfrak{p}(s+t)$ in $\mathfrak{D}(r+s+t)$ with respect to the Killing-Cartan form of $O(r+s+t)$. $C^{\infty}$-fiber bundle homomorphism $\bar{\varphi}: G_{r}\left(\varphi^{-1} \mathcal{E}\right) \rightarrow G_{r}(\mathcal{E})$ maps each fiber of $G_{r}\left(\varphi^{-1} \mathcal{E}\right)$ onto that of $G_{r}(\mathcal{E})$ 
so that the differential $d \bar{\varphi}: T G_{r}\left(\varphi^{-1} \mathcal{E}\right) \rightarrow T G_{r}(\mathcal{E})$ maps $\operatorname{Ker} d \pi_{r(\varphi-1 \mathcal{E})}$ to $\operatorname{Ker} d \pi_{G_{r}(\mathcal{E})}$. Let $(d \bar{\varphi})^{\mathscr{Q}}: \operatorname{Ker} d \pi_{G_{r}\left(\varphi^{-1 \mathcal{E}}\right)} \rightarrow \operatorname{Ker} d \pi_{G_{r}(\mathcal{E})}$ be the restriction of $d \bar{\varphi}$ to $\operatorname{Ker} d \pi_{G_{r}(\varphi-1 \mathcal{E})}$. Then ${ }^{\mathcal{E}} \boldsymbol{I} \circ(d \bar{\varphi})^{\mathcal{C}}=\bar{\varphi}_{\kappa}{ }^{\circ} \varphi^{-1} \mathcal{E} \boldsymbol{I}$.

The induced linear homomorphisms between $C^{\infty}$-sections are denoted by ${ }^{\mathcal{E}} \boldsymbol{I}$, $\varphi^{-1} \hat{c} \boldsymbol{I}, \bar{\varphi}_{\kappa}$ and $(d \bar{\varphi})^{\omega}$.

Let $\left(\mathcal{E}, h_{\mathcal{E}},{ }^{\varepsilon} \nabla,{ }^{\mathcal{E}} \nabla \omega\right)$ be a system of a $C^{\infty}$-vector bundle with a fiber metric $h_{\mathcal{E}}$, a covariant differentiation ${ }^{\varepsilon} \nabla$ compatible to $h$ and the connection form ${ }^{\varepsilon} \nabla_{\omega}$ of ${ }^{\varepsilon} \nabla$. The induced system via $\varphi$ is denoted by $\left(\varphi^{-1} \mathcal{E},{ }^{\varphi} h_{\mathcal{E}},{ }^{\varphi}\left({ }^{\mathcal{E}} \nabla\right),{ }^{\left({ }^{\mathcal{E}} \nabla\right)} \omega\right)$. Notice that

$$
\begin{aligned}
& (d \bar{\varphi})_{\varphi-1} \mathcal{E}_{\zeta(\tilde{E})}\left(\operatorname{Ker}^{\varphi\left(\mathcal{E}^{\nabla}\right)} \boldsymbol{\omega}_{\varphi-1} \mathcal{E}_{\zeta(\tilde{E})}\right) \subset \operatorname{Ker}^{\mathcal{E} \nabla} \omega_{\bar{\varphi}(\varphi-1 \mathcal{E} \zeta(\tilde{E}))} \text { for any } \tilde{E} \in O\left(\varphi^{-1} \mathcal{E},{ }^{\varphi} h_{\mathcal{E}}\right) \\
& \text { and }(d \bar{\varphi})^{\mathscr{V}} \circ\left(d \gamma_{\varphi-1 \mathscr{I}}\right)^{V}=\left(d \gamma_{\mathscr{I}}\right)^{V} \circ d \varphi \text {. }
\end{aligned}
$$

PROPOSITION 4.1.

$$
\left(d \gamma_{F}\right)_{\varphi(x)}^{V}\left((d \varphi)_{x} T_{x} M\right)=0 \quad \text { for all } x \in M
$$

if and only if $\gamma_{\varphi-1 \mathcal{F}}$ is a horizontal section with respect to ${ }^{\varphi}\left({ }^{\varepsilon} \nabla\right)$.

In the case of $L=P \cdot P^{-1}, \mathscr{F}=\operatorname{Ker} d \pi_{P \cdot P-1}$ and $\varphi=\varepsilon$, we have

COROLLARY 4.2 .

$$
\left(d \gamma_{P \cdot P-1}\right)_{\varepsilon(x)}^{V}\left((d \varepsilon)_{x} T_{x} M\right)=0 \quad \text { for all } x \in M
$$

if and only if ${ }^{\varepsilon} \gamma_{P \cdot P-1}$ is a horizontal section with respect to ${ }^{\varepsilon}\left({ }^{P \cdot P-1} \nabla\right)$.

From Proposition 3.6 and the above corollary, we have

THEOREM 4.3. $\omega$ is flat if and only of $\varepsilon_{P \cdot P-1}$ is a horizontal section with respect to ${ }^{\varepsilon}\left(P^{P \cdot P-1} \nabla\right)$.

Henceforth let $\left(M, 2^{M} g\right)$ be a Riemannian manifold. Proposition 3.6 implies that:

PROPOSITION 4.4 .

$$
\boldsymbol{e}^{V}\left({ }^{\varepsilon} \gamma_{P \cdot P-1}\right)(x)=\frac{1}{2} \boldsymbol{e}^{V}\left(\gamma_{P \cdot P-1}\right)(\varepsilon(x)) \quad \text { for any } x \in M
$$

Let ${ }^{L} g$ be a Riemmanian metric on $L . G_{r}(\mathcal{E})$ (resp. $G_{r}\left(\varphi^{-1} \mathcal{E}\right)$ ) has the horizontally lifted metric by ${ }^{\varepsilon} \nabla \omega$ (resp. ${ }^{\varphi\left(\varepsilon_{\nabla}\right)} \omega$ ) and its Riemannian connection $\nabla$ (resp. ${ }^{\varphi} \nabla$ ). Let $\nabla^{V}$ (resp. ${ }^{\varphi} \nabla^{V}$ ) be the induced connection in $\operatorname{Ker} d \pi_{G_{r}(\mathcal{E})}$ (resp.

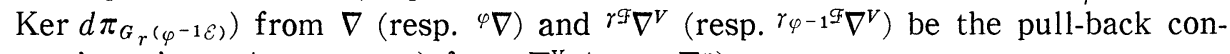
nection via $\gamma_{\mathcal{I}}\left(\right.$ resp. $\left.\gamma_{\varphi-1 \mathscr{T}}\right)$ from $\nabla^{V}$ (resp. $\left.\nabla^{\varphi}\right)$.

Note that $\varphi^{\varphi}\left(\gamma^{\mathcal{E}}\left({ }^{\mathcal{E}} \boldsymbol{I}\right)\right)={ }^{\gamma}{ }_{\varphi-1}{ }^{\mathscr{T}}\left(\bar{\varphi}^{\mathcal{C}}\left({ }^{\mathcal{I}} \boldsymbol{I}\right)\right)$ is connection-preserving [12, Theorem 8(2)]. Let $\gamma_{\varphi-1}{ }^{\mathscr{I}}(d \bar{\varphi})^{\mathcal{Q}}$ be the induced linear isomorphism between $C^{\infty}$-sections via $\gamma_{\varphi^{-1 q}}$. 


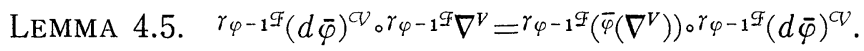

By a straightforward computation using the above lemma, we have the following formulas analogous to [2, Proposition 2.20]:

PROPOSITION 4.6.

(i) $r_{\varphi-1}{ }^{\mathscr{T}}(d \bar{\varphi})^{\mathscr{Q}}\left(r_{\varphi-1}{ }^{\mathscr{T}} \nabla^{V}\left(d \gamma_{\varphi-1 q}\right)^{V}(X, Y)\right)$

$$
\left.=\left(d \gamma_{\mathcal{I}}\right)^{V}\left({ }^{\varphi}\left({ }^{L} \nabla\right) d \varphi\right)_{x}(X, Y)\right)+{ }^{\gamma^{\mathscr{T}}} \nabla^{V}\left(d \gamma_{\mathcal{F}}\right)^{V}\left((d \varphi)_{x} X,(d \varphi)_{x} Y\right)
$$

for any $x \in M, X, Y \in T_{x} M$, where ${ }^{L} \nabla$ is the Riemmanian connection of ${ }^{L} g$.

(ii) $\gamma_{\varphi-1} \mathcal{I}(d \bar{\varphi})^{\mathcal{V}}\left(\tau^{V}\left(\gamma_{\varphi-1 \mathscr{F}}\right)_{x}\right)$

$$
=\left(d \gamma_{\mathcal{F}}\right)_{x}^{V}\left(\tau(\varphi)_{x}\right)+\sum_{\imath=1}^{m}{ }^{\gamma \mathscr{I}} \nabla^{V}\left(d \gamma_{\mathcal{F}}\right)^{V}\left((d \varphi)_{x} E_{\imath},(d \varphi)_{x} E_{\imath}\right)
$$

for any $x \in M$, where $\left\{E_{\imath}\right\}_{\imath=1}^{m}$ is an orthonormal basis for $\left(T_{x} M, 2^{M} g\right)$.

Proof. (i) Extend $X$ and $Y$ to local vector fields. The above lemma implies that

$$
\begin{aligned}
& \gamma_{\varphi-1}{ }^{\mathscr{I}}(d \bar{\varphi})^{\mathscr{Q}}\left(r_{\varphi-1^{\mathscr{I}}} \nabla^{V}\left(d \gamma_{\varphi-1 \mathscr{I}}\right)^{V}(X, Y)\right)
\end{aligned}
$$

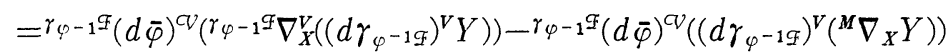

$$
\begin{aligned}
& =\varphi^{-1}{ }^{\Phi} \nabla_{X}^{V}\left(\left(d \gamma_{\mathscr{F}}\right)^{V}((d \varphi) Y)\right)-\left(d \gamma_{\mathcal{F}}\right)^{V}\left((d \varphi)\left({ }^{M} \nabla_{X} Y\right)\right) \\
& ={ }^{\varphi-1}{ }^{\mathscr{}} \nabla_{X}^{V}\left(d \gamma_{\mathcal{I}}\right)^{V}((d \varphi) Y)+\left(d \gamma_{\mathcal{F}}\right)^{V}\left({ }^{\varphi}\left({ }^{L} \nabla\right)_{X}((d \varphi) Y)\right)-\left(d \gamma_{\mathcal{F}}\right)^{V}\left((d \varphi)\left({ }^{M} \nabla_{X} Y\right)\right) \\
& ={ }^{\gamma^{\Phi}} \nabla_{(d \varphi) X}^{V}\left(d \gamma_{\mathcal{F}}\right)^{V}((d \varphi) Y)+\left(d \gamma_{\mathcal{F}}\right)^{V}\left({ }^{\varphi}\left({ }^{L} \nabla\right)_{X}((d \varphi) Y)-(d \varphi)\left({ }^{M} \nabla_{X} Y\right)\right) \\
& ={ }^{r^{q}} \nabla^{V}\left(d \gamma_{\mathcal{F}}\right)^{V}((d \varphi) X,(d \varphi) Y)+\left(d \gamma_{\mathcal{F}}\right)^{V}\left(\left(\varphi^{-1} L \nabla d \varphi\right)(X, Y)\right) \text {. }
\end{aligned}
$$

(ii) It follows from (i).

COROLLARY 4.7. If $\varphi$ is totally geodesic, then

(i) $\left.\gamma_{\varphi-1}{ }^{\mathscr{F}}(d \bar{\varphi})^{\mathscr{V}}\left(\gamma_{\varphi-1}{ }^{\mathscr{I}} \nabla^{V}\left(d \gamma_{\varphi-1 \mathscr{I}}\right)^{V}(X, Y)\right)={ }^{\mathscr{I}} \nabla^{V}\left(d \gamma_{\mathcal{F}}\right)^{V}\left((d \varphi)_{x} X,(d \varphi)_{x} Y\right)\right)$

for any $x \in M, X, Y \in T_{x} M$,

(ii) $r_{\varphi-1}{ }^{\mathscr{G}}(d \bar{\varphi})^{\mathscr{V}}\left(\tau^{V}\left(\gamma_{\varphi-1 \mathcal{F}}\right)_{x}\right)=\sum_{\imath=1}^{m} \gamma^{\mathscr{T}} \nabla^{V}\left(d \gamma_{\mathcal{I}}\right)^{V}\left((d \varphi)_{x} E_{\imath},(d \varphi)_{x} E_{\imath}\right)$

for any $x \in M$.

Even if $\varphi$ is totally geodesic, vertical harmonicity of $\gamma_{\varphi-1 \Phi}$ generally fails to inherit from that of $\gamma_{\mp}$. But an exceptional success lies in the case of $\varphi=\varepsilon$, $\mathscr{I}=\operatorname{Ker} d \pi_{P \cdot P-1}$. 
PROPOSITION 4.8.

(i) $\left.{ }^{\varepsilon} \gamma_{P \cdot P^{-1}}(d \bar{\varepsilon})^{\mathscr{C}}\left({ }^{\varepsilon} \gamma_{P \cdot P^{-1}} \nabla^{V}\left(d^{\varepsilon} \gamma_{P \cdot P-1}\right)^{V}(X, Y)\right)={ }^{\gamma P \cdot P^{-1}} \nabla^{V}\left(d \gamma_{P \cdot P-1}\right)^{V}(d \varepsilon)_{x} X,(d \varepsilon)_{x} Y^{\gamma}\right)$ for any $x \in M, X, Y \in T_{x} M$.

(ii) ${ }^{\varepsilon} \gamma_{P \cdot P^{-1}}(d \bar{\varepsilon})^{\mathcal{V}}\left(\tau^{V}\left({ }^{\varepsilon} \gamma_{P \cdot P^{-1}}\right)_{x}\right)=\sum_{\imath=1}^{m}{ }^{\gamma \cdot P^{-1}} \nabla^{V}\left(d \gamma_{P \cdot P-1}\right)^{V}\left((d \varepsilon)_{x} E_{\imath},(d \varepsilon)_{x} E_{\imath}\right)$ for any $x \in M$ where $\left\{E_{\imath}\right\}_{\imath=1}^{m}$ is an orthonormal basis for $\left(T{ }_{x} M, 2^{M} g\right)$.

COROLLARY 4.9 .

$$
{ }^{\gamma_{P \cdot P-1}} \nabla^{V}\left(d \gamma_{P \cdot P-1}\right)^{V}\left((d \varepsilon)_{x} T_{x} M,(d \varepsilon)_{x} T_{x} M\right)=0 \quad \text { for all } x \in M
$$

if and only if ${ }^{\varepsilon} \gamma_{P \cdot P-1}$ is a covariantly horizontal section with respect to ${ }^{\varepsilon}\left({ }^{P \cdot P-1} \nabla\right)$.

THEOREM 4.10. $\omega$ is parallel if and only if ${ }^{\varepsilon} \gamma_{P \cdot P-1}$ is a covariantly hortzontal section with respect to ${ }^{\varepsilon}\left({ }^{P \cdot P-1} \nabla\right)$.

COROLLARY 4.11.

$$
\left.\sum_{\imath=1}^{m}{ }^{\gamma_{P \cdot P-1}} \nabla^{V}\left(d \gamma_{P \cdot P-1}\right)^{V}\left((d \varepsilon)_{x} E_{\imath},(d \varepsilon)_{x} E_{\imath}\right)\right)=0 \quad \text { for all } x \in M
$$

if and only if ${ }^{8} \gamma_{P \cdot P-1}$ is a harmonic section with respect to ${ }^{\varepsilon}\left({ }^{P \cdot P-1} \nabla\right)$.

THEOREM 4.12. $\omega$ is Yang-Mills if and only if ${ }^{\varepsilon} \gamma_{P \cdot P-1}$ is a harmonic section with respect to ${ }^{\varepsilon}\left({ }^{P \cdot P-1} \nabla\right)$.

\section{§5. Reduction of Target Fibers}

Let $H$ be a Lie group which admits a bi-invariant metric and $H_{0}$ be a closed subgroup of $H$. We consider a right principal $H$-bundle $\mathscr{P}$ over a Riemannian manifold $M$, a principal $H_{0}$-subbundle $Q$, a reduction map of structure group $\overline{\boldsymbol{i}}: Q \rightarrow \mathscr{Q}$ and a right connection form $\overline{\boldsymbol{\omega}}$ which is reducible with respect to $\overline{\boldsymbol{i}}$. The $\bar{\omega}$ - (resp. $i^{*} \bar{\omega}-$ ) horizontally lifted metric on $\mathscr{Q}$ (resp. $Q$ ) is denoted by ${ }_{\bar{\omega}}^{\mathscr{Q}} g$ (resp. $\underset{i * \bar{\omega}}{Q} g$ ).

PROPOSITION 5.1.

( i ) $(d \overline{\boldsymbol{i}})_{v} S^{V}=(d \overline{\boldsymbol{i}})_{v}^{V} S,(d \overline{\boldsymbol{i}})_{v} S^{H}=(d \overline{\boldsymbol{i}})_{v}^{H} S$ for any $v \in Q, S \in T_{v} Q$.

(ii) $\bar{i}:\left(Q, \frac{Q}{i * \bar{\omega}} g\right) \rightarrow\left(\mathscr{P}, \frac{\mathscr{P}}{\bar{\omega}} g\right)$ is an isometric embedding.

(iii) $\left({ }^{i} \nabla d \bar{i}\left(S, T^{v}\right)\right)^{v}=0$, for all $v \in Q, S, T \in T_{v} Q$.

Proof. (i), (ii) trivial. (iii) Extend $S$ and $T$ to local vector fields. The restriction of $\overline{\boldsymbol{i}}$ to each fiber of $Q$ is a totally geodesic embedding into a fiber 
of $\mathscr{P}$ so that $\left({ }^{i} \nabla d \overline{\boldsymbol{i}}\left(S^{V}, T^{V}\right)\right)^{V}=0$. On the other hand, from (i) and [12, Lemma $1.4]$,

$$
\begin{aligned}
& \overline{\boldsymbol{\omega}}\left({ }^{i} \nabla d \overline{\boldsymbol{i}}\left(S^{H}, T^{V}\right)\right)=\overline{\boldsymbol{\omega}}\left({ }^{\Phi} \nabla_{(d \boldsymbol{i}) S^{H}}(d \overline{\boldsymbol{i}}) T^{V}-(d \overline{\boldsymbol{i}})\left({ }^{\complement} \nabla_{S H} T^{V}\right)\right) \\
& ={ }^{\mathscr{P}} d_{(d \overline{\boldsymbol{i}}) S^{H}}\left(\overline{\boldsymbol{\omega}}(d \overline{\boldsymbol{i}}) T^{V}\right)-\overline{\boldsymbol{\omega}}\left((d \overline{\boldsymbol{i}})^{Q} \nabla_{S^{H}} T^{V}\right)={ }^{\boldsymbol{i}}\left({ }^{\mathscr{P}} d\right)_{S H}\left(\left(\overline{\boldsymbol{i}}^{*} \overline{\boldsymbol{\omega}}\right) T^{V}\right)-\left(\overline{\boldsymbol{i}}^{*} \boldsymbol{\omega}\right)\left({ }^{C} \nabla_{S^{H}} T^{V}\right) \\
& { }^{Q} d_{S^{H}}\left(\left(\overline{\boldsymbol{i}}^{*} \overline{\boldsymbol{\omega}}\right) T^{V}\right)-{ }^{Q} d_{S H}\left(\left(\overline{\boldsymbol{i}}^{*} \overline{\boldsymbol{\omega}}\right) T^{V}\right)=0 .
\end{aligned}
$$

Let $H_{1}$, be another closed subgroup of $H$ and $Q / H_{0} \cap H_{1}, \mathscr{Q} / H_{1}$ be the $H_{0} \cap H_{1}$ orbit spase of $Q$, the $H_{1}$-orbit space of $\mathscr{P}$, respectively. The canonical quotient maps are denoted by $\pi_{/ H_{0} \cap H_{1}}: Q \rightarrow Q / H_{0} \cap H_{1}, \pi_{/ H_{1}}: \mathscr{Q} \rightarrow \mathscr{Q} / H_{1}$. $\overline{\boldsymbol{i}}$ is right $H_{0^{-}}$ (therefore $H_{0} \cap H_{1}-$ ) equivariant so that there uniquely exists $i: Q / H_{0} \cap H_{1} \rightarrow \mathscr{Q} / H_{1}$ such that $\boldsymbol{i} \circ \pi_{/ H_{0} \cap H_{1}}=\pi_{/ H_{1}} \circ \bar{i}$.

Note that $Q_{/ H_{0} \cap H_{1}}, \mathscr{P}_{/ H_{1}}$ is associated to $Q, \mathscr{P}$, and let ${ }_{i * \bar{\omega}}^{Q / H_{0} \cap H_{1}} g, \frac{\mathscr{P}}{\bar{\omega}} / H_{1} g$ be the horizontally lifted metrics on $Q / H_{0} \cap H_{1}, \mathscr{Q} / H_{1}$ by $\overline{\boldsymbol{i}}^{*} \bar{\omega}, \bar{\omega}$, respectively.

PROPOSITION 5.2.

(i) $(d i)_{\pi / H_{0} \cap H_{1}(v)} Z^{V}=(d i)_{\pi / H_{0} \cap H_{1}(v)}^{V} Z,(d i)_{\pi / H_{0} \cap H_{1}(v)} Z^{H}=(d i)_{\pi / H_{0} \cap H_{1}(v)}^{H} Z$, for any $v \in Q, Z \in T_{\pi / H_{0} \cap H_{1}(v)}\left(Q / H_{0} \cap H_{1}\right)$.

(ii) $\quad i:\left(Q / H_{0} \cap H_{1}, \stackrel{Q / H^{\prime} H_{0} \cap H_{1}}{g}\right) \rightarrow\left(\mathscr{Q} / H_{1}, \frac{\mathscr{Q}}{\bar{\omega}} / H_{1} g\right)$ is an isometric embedding.

These metrics make $\pi_{/ H_{0} \cap H_{1}}:(Q, \underset{i * \bar{\omega}}{Q} g) \rightarrow\left(Q / H_{0} \cap H_{1}, \underset{i * \bar{\omega}}{Q / H} H_{0 \cap H_{1}} g\right)$ and $\pi_{/ H_{1}}:$ $\left(\mathscr{P}, \frac{\mathscr{P}}{\bar{\omega}} g\right) \rightarrow\left(\mathscr{Q} / H_{1}, \frac{\mathscr{P}}{\overline{\bar{\omega}}} / H_{1} g\right)$ into Riemannian submersions with totally geodesic fibers. We write the associated orthogonal splittings as follows:

$$
\begin{aligned}
& T Q=\operatorname{Ker} d \pi_{/ H_{0} \cap H_{1}} \oplus\left(\operatorname{Ker} d \pi_{/ H_{0} \cap H_{1}}\right)^{\perp} ; S=S_{C \mathcal{V}}+S_{\mathscr{H}}, \\
& T \mathscr{Q}=\operatorname{Ker} d \pi_{/ H_{1}} \oplus\left(\operatorname{Ker} d \pi_{/ H_{1}}\right)^{\perp} ; V=V_{\mathcal{V}}=V_{\mathscr{H}} .
\end{aligned}
$$

\section{PROPOSITION 5.3.}

$$
(d \overline{\boldsymbol{i}})_{v} S_{Q}=\left((d \overline{\boldsymbol{i}})_{v} S\right)_{\mathscr{V}}, \quad(d \overline{\boldsymbol{i}})_{v} S_{\mathscr{H}}=\left((d \overline{\boldsymbol{i}})_{v} S\right)_{\mathscr{H}}, \quad \text { for all } v \in Q, S \in T_{v} Q .
$$

We denote the horizontal lifts of $Z \in T\left(Q / H_{0} \cap H_{1}\right), U \in T\left(\mathscr{P} / H_{1}\right)$, by $\mathscr{H} Z$, $\mathscr{H} U$, respectively.

PROPOSITION 5.4.

(i) $\mathscr{H} Z^{V}=(\mathscr{H} Z)^{V}, \mathscr{H} Z^{H}=(\mathscr{H} Z)^{H}$, for all $v \in Q, Z \in T_{\pi / H_{0} \cap H_{1}(v)}\left(Q / H_{0} \cap H_{1}\right)$.

(ii) $\mathscr{H} U^{V}=(\mathscr{H} U)^{V}, \mathscr{H} U^{H}=(\mathscr{H} U)^{V}$, for all $u \in \mathscr{P}, U \in T_{\pi / H_{1}(u)}\left(\mathscr{P} / H_{1}\right)$.

(iii) $\mathscr{H}(d \boldsymbol{i})_{\pi / H_{0} \cap H_{1}(v)} W^{V}=(d \overline{\boldsymbol{i}})_{v} \mathscr{H} W^{V}, \mathscr{H}(d \boldsymbol{i})_{\pi / H_{0} \cap H_{1}(v)} W^{H}=(d \overline{\boldsymbol{i}})_{v} \mathscr{H} W^{H}$, for all $v \in Q, W \in T_{\pi / H_{0} \cap H_{1}(v)}\left(Q / H_{0} \cap H_{1}\right)$. 
PROPOSITION 5.5.

$\left({ }^{i} \nabla d \boldsymbol{i}\left(Z, W^{V}\right)\right)^{V}=0, \quad$ for all $v \in Q, Z, W \in T_{\pi / H_{0} \cap H_{1}(v)}\left(Q / H_{0} \cap H_{1}\right)$.

Proof. Extend $Z, W$ to local vector fields. From Propositions 5.1, 5.3, 5.4 and [9, Lemma 1],

$$
\begin{aligned}
\mathscr{H}\left({ }^{\boldsymbol{i}} \nabla\right. & \left.d \boldsymbol{i}\left(Z, W^{V}\right)\right)^{V}=\left(\mathscr{H}^{\mathscr{P} / H_{1}} \nabla_{(d \boldsymbol{i}) Z}(d \boldsymbol{i}) W^{V}\right)^{V}-\left(\mathscr{H}(d \boldsymbol{i})\left({ }^{Q / H_{0} \cap H_{1}} \nabla_{Z} W^{V}\right)\right)^{V} \\
& =\left(\left({ }^{\mathscr{P}} \nabla_{\mathscr{H}(d \boldsymbol{i}) Z} \mathscr{H}(d \boldsymbol{i}) W^{V}\right)_{\mathscr{H}}\right)^{V}-\left((d \boldsymbol{i}) \mathscr{H}\left({ }^{Q / H_{0} \cap H_{1}} \nabla_{Z} W^{V}\right)\right)^{V} \\
& =\left(\left({ }^{\mathscr{P}} \nabla_{(d \boldsymbol{i}) \mathscr{H} Z}(d \overline{\boldsymbol{i}}) \mathscr{H} W^{V}\right)_{\mathscr{H}}\right)^{V}-\left((d \overline{\boldsymbol{i}})\left({ }^{Q} \nabla_{\mathscr{H} Z} \mathscr{H} W^{V}\right)_{\mathscr{H}}\right)^{V} \\
& =\left(\left({ }^{\mathscr{P}} \nabla_{(d \boldsymbol{i}) \mathscr{H} Z}(d \overline{\boldsymbol{i}}) \mathscr{H} W^{V}-(d \overline{\boldsymbol{i}})^{\complement} \nabla_{\mathscr{H} Z} \mathscr{H} W^{V}\right)^{V}\right)_{\mathscr{H}} \\
& =\left(\left(\left({ }^{i} \nabla d \boldsymbol{i}\left(\mathscr{H} Z, \mathscr{H} W^{V}\right)\right)^{V}\right)_{\mathscr{H}}=0_{\mathscr{H}}=0 .\right.
\end{aligned}
$$

Let $\gamma_{0}: M \rightarrow Q / H_{0} \cap H_{1}$ be a $C^{\infty}$-section (if exists). $\quad \gamma_{1}=\boldsymbol{i} \circ \gamma_{0}: M \rightarrow \mathscr{Q} / H_{1}$ is also a $C^{\infty}$-section.

PROPOSITION 5.6.

(i ) $\left(d \gamma_{1}\right)^{V} Y=(d \boldsymbol{i})_{\gamma_{0}(x)}\left(\left(d \gamma_{0}\right)^{V} Y\right)$, for all $x \in M, Y \in T_{x} M$.

(ii) $\boldsymbol{e}^{V}\left(\gamma_{1}\right)=\boldsymbol{e}^{V}\left(\gamma_{0}\right)$.

(iii) ${ }^{r_{1}} \nabla^{V}\left(d \gamma_{1}\right)^{V}(X, Y)=(d i)_{\gamma_{0}(x)}\left({ }^{\circ} \nabla^{V}\left(d \gamma_{0}\right)^{V}(X, Y)\right)$ for all $x \in M, X, Y \in T_{x} M$.

(iv) $\tau^{V}\left(\gamma_{1}\right)_{x}=(d i)_{r_{0}(x)}\left(\tau^{V}\left(\gamma_{0}\right)_{x}\right)$ for all $x \in M$.

Proof. (i) It follows from Proposition 5.2 (i). (ii) From (i) and Proposition 5.2 (ii). (iii) Extend $X, Y$ to local vector fields. From (i) and Proposition 5.5 ,

$$
\begin{aligned}
r_{1} \nabla^{V}\left(d \gamma_{1}\right)^{V}(X, Y)=\left({ }^{\mathscr{P} / H_{1}} \nabla_{\left(d \gamma_{1}\right) X}\left(d \gamma_{1}\right)^{V} Y-\left(d \gamma_{1}\right)\left({ }^{M} \nabla_{X} Y\right)\right)^{V} \\
=\left({ }^{\mathscr{P} / H_{1}} \nabla_{(d \boldsymbol{i})\left(d \gamma_{0}\right) X}(d \boldsymbol{i})\left(d \gamma_{0}\right)^{V} Y\right)^{V}-\left((d \boldsymbol{i})\left(d \gamma_{0}\right)^{V}\left({ }^{M} \nabla_{X} Y\right)\right)^{V} \\
=\left({ }^{i} \nabla_{\left(d \gamma_{0}\right) X}(d \boldsymbol{i})\left(d \gamma_{0}\right)^{V} Y\right)^{V}-\left((d \boldsymbol{i})\left({ }^{Q / H_{0} \cap H_{1}} \nabla_{\left(d \gamma_{0}\right) X}\left(d \gamma_{0}\right)^{V} Y\right)\right)^{V} \\
\quad+\left((d \boldsymbol{i})\left({ }^{Q / H_{0} \cap H_{1}} \nabla_{\left(d \gamma_{0}\right) X}\left(d \gamma_{0}\right)^{V} Y\right)\right)^{V}-(d \boldsymbol{i})\left(d \gamma_{0}\right)^{V}\left({ }^{M} \nabla_{X} Y\right) \\
={ }^{i} \nabla d \boldsymbol{i}\left(\left(d \gamma_{0} X,\left(d \gamma_{0}\right)^{V} Y\right)+d \boldsymbol{i}\left({ }^{\gamma} \nabla^{V}\left(d \gamma_{0}\right)^{V}(X, Y)\right)=d \boldsymbol{i}\left(r_{0} \nabla^{V}\left(d \gamma_{0}\right)^{V}(X, Y)\right) .\right.
\end{aligned}
$$

(iv) It follows from (iii).

COROLLARY 5.7. $\gamma_{0}$ is a horizontal, covarantly horizontal or harmonic section if and only if $\gamma_{1}$ is a horizontal, covariantly horizontal or harmonic section, respectively.

Let $\left(\mathcal{E}, h_{\mathcal{E}}\right), O\left(\mathcal{E}, h_{\mathcal{E}}\right)$ be a system of a $C^{\infty}$-vector bundle of rank $r+s+t$ 
and the orthonormal frame bundle of $\left(\mathcal{E}, h_{\mathcal{E}}\right)$, whose structure group is $O(r+s+t)$. For a vector subbundle $\mathcal{S}$ of rank $r+s$, the inclusion is denoted by $i_{\mathcal{S}}^{\mathcal{E}}: \mathcal{S} \rightarrow \mathcal{E}$. The orthogonal splitting $\mathcal{E}=\mathcal{S} \oplus \mathcal{S}^{\perp}$ induces the adapted (c. f. [7]) orthonormal frame bundle $O\left(\mathcal{S} \oplus \mathcal{S}^{\perp}, h_{S} \oplus h_{S_{\perp}}\right)$, whose structure group is $O(r+s)$ $\times O(t)$, where $h_{\mathcal{S}}, h_{\mathcal{S}}$ are the restrictions of $h_{\mathcal{E}}$ to $\mathcal{S}, \mathcal{S}^{\perp}$, respectively. The inclusion ${ }^{o} i_{\mathcal{S}}: O\left(\mathcal{S} \oplus \mathcal{S}^{\perp}, h_{\mathcal{S}} \oplus h_{\mathcal{S}}\right) \rightarrow O\left(\mathcal{E}, h_{\mathcal{E}}\right)$ is right $O(r+s) \times O(t)$-equi-variant.

$$
O(r+s) \times O(t) \cap O(r) \times O(s+t)=O(r) \times O(s) \times O(t)
$$

so that ${ }^{o} i_{S}^{\mathcal{E}}$ is reduced to the inclusion ${ }^{G r} i_{S}^{\mathcal{E}}: G_{r}(\mathcal{S}) \rightarrow G_{r}(\mathcal{E})$ which assigns $r$-plane $\mathscr{F}_{x}$ in $\mathcal{S}_{x}$ to $i_{S} \mathcal{E}_{x}$ in $\mathcal{E}_{x}$ for $x \in M$. A vector subbundle $\mathscr{I}$ of $\mathcal{S}$ (if exists) defines the Gauss sections :

$$
\gamma_{\Phi}^{\mathcal{S}}: M \rightarrow G_{r}(\mathcal{S}) ; x \mapsto \mathscr{F}_{x}, \gamma_{\Phi}^{\mathcal{E}}: M \rightarrow G_{r}(\mathcal{E}) ; x \mapsto i_{S}^{\mathcal{E}} \mathscr{F}_{x} .
$$

Let ${ }^{\varepsilon} \nabla$ be a connection in $\mathcal{E}$ compatible with $h_{\mathcal{E}}$, which preserves all $C^{\infty}$ sections of $\mathcal{S}$ (therefore $\mathcal{S}^{\perp}$ ).

PROPOSITION 5.8. $\boldsymbol{e}^{V}\left(\boldsymbol{\gamma}_{\mathscr{q}}^{\mathcal{S}}\right)=\boldsymbol{e}^{V}\left(\gamma_{\mathscr{q}}^{\mathcal{E}}\right)$

\section{PROPOSITION 5.9.}

$\gamma_{9}^{S}$ is a horizontal, covariantly horizontal or harmonic section with respect to ${ }^{s} \nabla$ if and only if

$\gamma_{\mathscr{F}}^{\mathcal{E}}$ is a horizontal, covariantly horizontal or harmonic section with respect to ${ }^{\varepsilon} \nabla$, respectively.

In the case of $r=p, s=t=m, \mathcal{E}=\varepsilon^{-1} T\left(P \cdot P^{-1}\right), \mathcal{S}=\varepsilon^{-1} \boldsymbol{E}^{(-)},{ }^{\mathcal{E}} \nabla={ }^{\varepsilon}\left(P^{P \cdot P-1} \nabla\right), \mathscr{F}=$ $\varepsilon^{-1} \operatorname{Ker} d \pi_{P \cdot P-1}, \gamma_{q}^{S}={ }^{\varepsilon} \gamma_{P \cdot P-1}^{(-)}$and $\gamma_{q}^{\mathcal{E}}={ }^{\varepsilon} \gamma_{P \cdot P-1}$, from Proposition 2.16 and [7, Chapter VII], we have the followings:

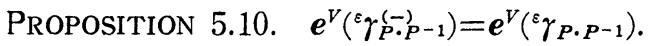

\section{PROPOSITION 5.11.}

${ }^{\varepsilon} \gamma_{P \cdot P-1}^{(-)}$is a horizontal, covariantly horizontal or harmonic section with respect to $\nabla^{(-)}$if and only if

${ }^{\varepsilon} \gamma_{P \cdot P-1}$ is a horizontal, covariantly horizontal or harmonic section with respect to ${ }^{E}\left({ }^{P \cdot P-1} \nabla\right)$, respectively.

By combining Theorems 4.3, 4.10, 4.12 and the above proposition and corollary, we obtain the main theorem:

THEOREM 5.12 (THEOREM D).

(i) $\frac{1}{2}\|\omega \Omega\|_{u}^{2}=\boldsymbol{e}^{V}\left(\gamma^{(-)}\right)(x)$ for all $x \in M, u \in \pi_{P}^{-1}(x)$.

(ii) $\omega$ is flat, parallel or Yang-Mills if and only if ${ }^{\varepsilon} \gamma_{P \cdot P-1}^{(-)}$is a horizontal, covariantly horizontal or harmonic section with respect to $\nabla^{(-)}$, respectively. 


\section{REFERENCES}

[1] J.P. Bourguignon, Harmonic curvature for gravitational and Yang-Mills fields, Lecture Notes in Math., 949, Springer, Berlin, 1982.

[2] J. Eellos and L. Lemaire, Selected Topics in Harmonic Maps, C. B. M.S. Regional Conference Series 50, American Mathematical Society, Providence, R.I., 1983.

[3] W. Greub, S. Halperin and R. Vanstone, Connections, Curvature and Cohomology, vol. 2, Academic Press, New York, 1973.

[4] M. Iтон, Invariant connections and Yang-Mills solutions, Trans. Amer. Math. Soc., 267 (1981), 229-236.

[5] S. Kobayashi, Theory of connections, Ann. Mat. Pura Appl. (4), 43 (1957), 119-194.

[6] S. Kobayashi and K. Nomizu, Foundations of Differential Geometry, vol. 1, Wiley, New York, 1969.

[7] S. kоваYASHi and K. Nomizu, Foundations of Differential Geometry, vol. 2, Wiley,New York, 1969.

[8] K. Mackenzie, Lie Groupoids and Lie Algebroids in Differential geometry, London Math. Soc. Lecture Note Series 124, Cambrige Univ. Press, 1987.

[9] B. O'NeILl, The fundamental equations of a submersion, Michigan Math. J., 13 (1966), 459-469.

[10] J. VILMs, Totally geodesic maps, J. Differential Geom., 4 (1970), 73-79.

[11] C.M. Wood, The Gauss sections of a Riemannian immersion, J. London Math. Soc. (2), 33 (1986), 157-168.

[12] C.M. Wood, Harmonic sections and Yang-Mills fields, Proc. London Math. Soc. (3), 54 (1987), 544-558.

Sasebo College of Technology

$1-1$, OKISHINCHO

Sasebo, Nagasaki, $857-11$

JAPAN 